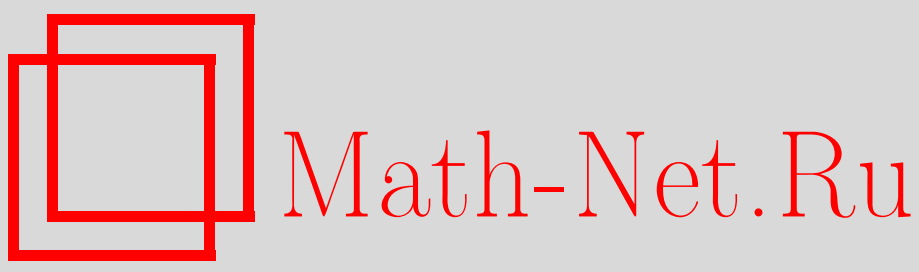

В. И. Буренков, А. Л. Горбунов, Точные оценки минимальной нормы операторов продолжения для пространств Соболева, Изв. РАН. Сер. матем., 1997, том 61, выпуск 1, 3-44

DOI: https://doi.org/10.4213/im103

Использование Общероссийского математического портала Math-Net.Ru подразумевает, что вы прочитали и согласны с пользовательским соглашением

http://www.mathnet.ru/rus/agreement

Параметры загрузки:

IP : 18.234 .156 .22

26 апреля 2023 г., 11:26:49 
УДК 517.518

В.И. Буренков, А. Л. Горбунов

\section{Точные оценки минимальной нормы операторов продолжения для пространств Соболева}

В статье рассматриваются операторы продолжения для пространств Соболева $W_{p}^{l}(\Omega)$, где $\Omega$ - область в $\mathbb{R}^{n}$ с границей класса Липшица. Для минимальной нормы оператора продолжения получена двухсторонняя оценка, точная по параметру гладкости $l$.

Библиография: 35 наименований.

\section{Введение}

В данной статье рассматриваются операторы продолжения

$$
T: W_{p}^{l}(\Omega) \rightarrow W_{p}^{l}\left(\mathbb{R}^{n}\right),
$$

действуюшие в пространстве Соболева $W_{p}^{l}(\Omega)$, где $n, l \in \mathbb{N}, 1 \leqslant p \leqslant+\infty$, $\Omega \subset \mathbb{R}^{n}$ - открытое множество класса Lip 1, состоящего из измеримых функций $f$, для которых имеет смысл и конечна норма

$$
\|f\|_{W_{p}^{l}(\Omega)}=\|f\|_{L_{p}(\Omega)}+\|f\|_{w_{p}^{l}(\Omega)},
$$

где

$$
\|f\|_{w_{p}^{l}(\Omega)}=\sum_{|\alpha|=l}\left\|D^{\alpha} f\right\|_{L_{p}(\Omega)}
$$

$\alpha=\left(\alpha_{1}, \ldots, \alpha_{n}\right)$ - мультииндекс, $\alpha_{i} \in \mathbb{N}_{0}, i=1, \ldots, n,|\alpha|=\alpha_{1}+\cdots+\alpha_{n}$, $D^{\alpha} f=D_{1}^{\alpha_{1}} \ldots D_{n}^{\alpha_{n}} f$ - обобщенная производная функции $f$.

Подробное изложение теории пространств Соболева дается в книгах [24], [34], [3]. Результаты изучения различных направлений задачи о продолжении функций из пространств Соболева можно найти в [19], [6], [34], [3], [15].

Предметом исследования в настоящей статье является оценка минимальной нормы операторов продолжения $T$ для пространств $W_{p}^{l}(\Omega)$ :

$$
\inf _{T: W_{p}^{l}(\Omega) \rightarrow W_{p}^{l}\left(\mathbb{R}^{n}\right)}\|T\|=\inf _{T: W_{p}^{l}(\Omega) \rightarrow W_{p}^{l}\left(\mathbb{R}^{n}\right)} \sup _{\substack{f \in W_{p}^{l}(\Omega) \\ f \neq 0}} \frac{\|T f\|_{W_{p}^{l}\left(\mathbb{R}^{n}\right)}}{\|f\|_{W_{p}^{l}(\Omega)}} .
$$

Получение двусторонних оценок минимальной нормы операторов продолжения, точных по тому или иному параметру, описывающему пространство $W_{p}^{l}(\Omega)$, представляет особый интерес, так как ее точное значение можно найти только в немногих случаях. Один из подобных случаев рассмотрен С.Г. Михлиным в [30], где автор находит точное значение наименьшей нормы оператора продолжения

$$
T: W_{2}^{1}\left(B_{1}\right) \rightarrow W_{2}^{1}\left(B_{\varrho}\right),
$$


где $B_{\varrho}$ - открытый шар радиуса $\varrho>0$.

В работах Паукшто М. В., Мазьи В.Г. и Поборчего С. В. получен ряд двусторонних оценок, точных по параметру, характеризующему область $\Omega$.

В [31] доказана двусторонняя, точная по параметру $\alpha$ оценка минимальной нормы оператора продолжения

$$
T: W_{2}^{1}\left(V_{\alpha}\right) \rightarrow W_{2}^{1}\left(B_{1}\right)
$$

где $V_{\alpha}=\left\{(r, \varphi) \in \mathbb{R}^{2}: 0<r<1,0<\varphi<\alpha\right\}, 0<\alpha<\frac{\pi}{2}$, имеющая следующий вид:

$$
C_{1} \alpha^{-\frac{1}{2}} \leqslant \inf _{T: W_{2}^{1}\left(V_{\alpha}\right) \rightarrow W_{2}^{1}\left(B_{1}\right)}\|T\| \leqslant C_{2} \alpha^{-\frac{1}{2}} .
$$

Константы $C_{1}$ и $C_{2}$ не зависят от $\alpha$.

Мазья В. Г. и Поборчий С. В. [20] вычислили двусторонние оценки минимальной нормы операторов продолжения

$$
T: W_{p}^{l}\left(\Omega_{\varepsilon}\right) \rightarrow W_{p}^{l}\left(B_{\varrho}\right),
$$

где $\Omega_{\varepsilon}=\left\{x \in \mathbb{R}^{n}: \frac{x}{\varepsilon} \in \Omega\right\}, \Omega$ - ограниченная липшищева область в $\mathbb{R}^{n}, B_{\varrho} \supset \Omega$, $\varepsilon \in\left(0, \frac{1}{2}\right), \varepsilon \leqslant \varrho \leqslant+\infty$. Норма в пространстве $W_{p}^{l}\left(\Omega_{\varepsilon}\right)$ выбирается в виде, отличном от $(1)$, а именно она содержит $L_{p}$-нормы всех производных до порядка $l$ включительно:

$$
\|f\|_{W_{p}^{l}\left(\Omega_{\varepsilon}\right)}=\sum_{0 \leqslant|\alpha| \leqslant l}\left\|D^{\alpha} f\right\|_{W_{p}^{l}\left(\Omega_{\varepsilon}\right)} .
$$

В [20] доказано, что

$$
\inf _{T: W_{p}^{l}\left(\Omega_{\varepsilon}\right) \rightarrow W_{p}^{l}\left(B_{\varrho}\right)}\|T\| \sim \begin{cases}\varepsilon^{-\frac{n}{p}} \min \left\{\varrho^{\frac{n}{p}}, 1\right\}, & \text { если } l p>n, \\ \varepsilon^{-\frac{n}{p}} \min \left\{\varrho^{\frac{n}{p}},\left|\log _{\varepsilon}\right|^{\frac{1-p}{p}}\right\}, & \text { если } l p=n, \\ \varepsilon^{-\frac{n}{p}} \min \left\{\varrho^{\frac{n}{p}}, \varepsilon^{\frac{n}{p}-l}\right\}, & \text { если } l p<n,\end{cases}
$$

где знак $\sim$ в выражении $A \sim B$ означает сушествование констант $C_{3}$ и $C_{4}$, зависящих только от $n, p, l, \Omega$ и не зависящих от $\varepsilon$ и $\varrho$ таких, что верно двустороннее неравенство $C_{3} B \leqslant A \leqslant C_{4} B$.

В работе [32] исследуется асимптотическое (по $l$ ) поведение норм операторов продолжения типа Стейна [34] $S: W_{2}^{l}\left(\mathbb{R}_{+}\right) \rightarrow W_{2}^{l}(\mathbb{R})$.

Целью настоящей статьи является получение двусторонней оценки минимальной нормы операторов продолжения для пространств Соболева (с нормой в виде (1)), точной по порядку гладкости $l$.

В $\S 1,2,4,5,6$ выводятся оценки элементов, определяющих оператор продолжения, который позволяет найти оценку сверху.

В $\S 1$ вычисляются в некотором смысле неулучшаемые оценки производных порядка не выше $l$ функций из класса $C_{0}^{\infty}\left(\mathbb{R}^{n}\right)$ с определенньми специальньми свойствами.

$\S 2$ посвящен оценкам ядер, входящих в интегральное представление функций. 
В одномерном случае для $\Omega=(a, b)$ в $\S 3$ приводится двусторонняя оценка минимальной нормы операторов продолжения, точная как по порядку гладкости $l$, так и по длине интервала $(a, b)$.

В $\S 4$ строится разбиение единицы и даются оценки производных порядка не выше $l$ от элементов разбиения единицы.

В $\S 5$ приводится двусторонняя оценка точной постоянной в неравенстве для $L_{p}$-нормы промежуточной производной, точная по порядку гладкости $l$.

В $\S 6$ доказываются оценки сверху нормы оператора продолжения, построенного Буренковым В.И. в [6], [11].

Основной результат статьи содержится в $\S 7$.

\section{§1. Теоремы об оценках производных финитных функций}

В этом параграфе строятся финитные бесконечно дифференцируемые ядра усреднения $\omega$ (т.е. $\omega \in C_{0}^{\infty}\left(\mathbb{R}^{n}\right)$ и $\int_{\mathbb{R}^{n}} \omega(x) d x=1$ ) с различными специальными свойствами и для них приводятся в определенном смысле неулучшаемые оценки производных.

Обозначим через $B(x, r)$ открытый шар в $\mathbb{R}^{n}$ радиуса $r$ с центром в точке $x$; $B_{r}:=B(0, r)$. Для любого множества $\Omega \in \mathbb{R}^{n}$ будем обозначать через $\bar{\Omega}$ его замыкание, а через $\Omega^{\delta}(\delta>0)$ его $\delta$-окрестность: $\Omega^{\delta}=\bigcup_{x \in \Omega} B(x, \delta)$.

Пусть $\delta>0$ и $\omega-$ ядро усреднения. Положим $\omega_{\delta}(x):=\frac{1}{\delta^{n}} \omega\left(\frac{x}{\delta}\right)$ и обозначим через $A_{\delta}$ оператор усреднения с шагом $\delta$ : для $f \in L_{1}^{\text {loc }}\left(\mathbb{R}^{n}\right)$

$$
\left(A_{\delta} f\right)(x)=\left(\omega_{\delta} * f\right)(x)=\frac{1}{\delta^{n}} \int_{\mathbb{R}^{n}} \omega\left(\frac{x-y}{\delta}\right) f(y) d y
$$

Отметим, что $A_{\delta} f \in C^{\infty}\left(\mathbb{R}^{n}\right)$ и

$$
\operatorname{supp} A_{\delta} f \subset \overline{(\operatorname{supp} f)^{\delta}} \text {. }
$$

\section{1. Неотрицательные ядра усреднений, функции типа “шапочки".}

Tеорема 1. а) Существует число $C_{1}>0$ и для любых $n, l \in \mathbb{N}$ существует функиия $\omega \in C_{0}^{\infty}\left(\mathbb{R}^{n}\right)$, удовлетворяющие следующим условиям:

1) $\operatorname{supp} \omega \subset B_{1}$

2) $\int_{\mathbb{R}^{n}} \omega(x) d x=1$

3) $\omega(x) \geqslant 0, \quad x \in \mathbb{R}^{n}$;

4) $\forall \alpha \in \mathbb{N}_{0}^{n}: \quad|\alpha| \leqslant l$

$$
\left\|D^{\alpha} \omega\right\|_{L_{\infty}\left(\mathbb{R}^{n}\right)} \leqslant 2^{n} v_{n}^{-1} C_{1}^{|\alpha| n} l^{|\alpha|},
$$

где $v_{n}=\pi^{\frac{n}{2}}\left(\Gamma\left(\frac{n}{2}+1\right)\right)^{-1}$ - обгем единичного шара в $\mathbb{R}^{n}$.

б) Для функиии $\omega \in C_{0}^{\infty}\left(\mathbb{R}^{n}\right)$, удовлетворяющей условиям 1)-3), нельзя подобрать числа $C_{2}>0$ и $\sigma<1$ такие, чтобь для любого $l \in \mathbb{N}$ выполнялось условие

$\left.4^{\prime}\right) \quad \forall \alpha \in \mathbb{N}_{0}^{n}:|\alpha| \leqslant l$

$$
\left\|D^{\alpha} \omega\right\|_{L_{\infty}\left(\mathbb{R}^{n}\right)} \leqslant C_{2}^{|\alpha|} l^{\sigma|\alpha|}
$$


ДоказАтельство. Докажем п.а). П.б) будет доказан в конце $\S 7$. Пусть $\chi_{1}=\frac{2^{n}}{v_{n}} \chi$, где $\chi$ - характеристическая функция множества $B_{\frac{1}{2}}$ и $g \in C_{0}^{\infty}\left(B_{1}\right)-$ ядро усреднения, $g \geqslant 0$. Рассмотрим функцию $\eta$,

$$
\eta(x)= \begin{cases}h(1-|x|), & |x| \leqslant 1 \\ 0, & |x|>1\end{cases}
$$

где $h=\left(\int_{B_{1}}(1-|x|) d x\right)^{-1}=\frac{(n+1)}{v_{n}}$.

Для $\delta=\frac{1}{2(l+1)}$ положим $\omega=\left(A_{\delta}^{l} \chi_{1}\right) * g_{\delta}$, где $A_{\delta}^{l}-l$-я степень оператора $A_{\delta} \mathrm{c}$ ядром $\eta$.

Согласно $(2) \operatorname{supp} \omega \subset \overline{\left(\ldots\left(\operatorname{supp} \chi_{1}\right)^{\delta} \ldots\right)^{\delta}}=\bar{B}_{\frac{1}{2}}$, что доказывает п. 1$)$.

П. 3) следует из неотрищательности ядра усреднения $g$.

Проверим п. 4). Пусть $\alpha \in \mathbb{N}_{0}^{n}:|\alpha|<l$; тогда согласно свойствам сверток

$$
\begin{aligned}
\left\|D^{\alpha} \omega\right\|_{L_{\infty}\left(\mathbb{R}^{n}\right)} & =\left\|D^{\alpha}\left(\chi_{1} * \eta_{\delta} * \cdots * \eta_{\delta} * g_{\delta}\right)\right\|_{L_{\infty}\left(\mathbb{R}^{n}\right)} \\
& =\left\|\left(D_{i_{1}} \eta_{\delta}\right) * \cdots *\left(D_{i_{|\alpha|} \mid} \eta_{\delta}\right) * \eta_{\delta} \ldots \eta_{\delta} * g_{\delta} * \chi_{1}\right\|_{L_{\infty}\left(\mathbb{R}^{n}\right)} .
\end{aligned}
$$

Из (1) и неравенства

$$
\|f * g\|_{L_{\infty}\left(\mathbb{R}^{n}\right)} \leqslant\|f\|_{L_{1}\left(\mathbb{R}^{n}\right)}\|g\|_{L_{\infty}\left(\mathbb{R}^{n}\right)}
$$

$(l+1)$ раз примененного к $(5)$, получаем следующее неравенство:

$$
\begin{aligned}
\left\|D^{\alpha} \omega\right\|_{L_{\infty}\left(\mathbb{R}^{n}\right)} & \leqslant 2^{n} v_{n}^{-1}\left\|D_{i_{1}} \eta_{\delta}\right\|_{L_{1}\left(\mathbb{R}^{n}\right)} \ldots\left\|D_{i_{|\alpha|}} \eta_{\delta}\right\|_{L_{1}\left(\mathbb{R}^{n}\right)}\left\|\eta_{\delta}\right\|_{L_{1}\left(\mathbb{R}^{n}\right)}^{l-|\alpha|}\left\|g_{\delta}\right\|_{L_{1}\left(\mathbb{R}^{n}\right)} \\
& =2^{n} v_{n}^{-1}\left\|D_{1} \eta_{\delta}\right\|_{L_{1}\left(\mathbb{R}^{n}\right)}^{|\alpha|}
\end{aligned}
$$

Здесь мы учли сферическую симметрию функции $\eta$ и тот факт, что $\left\|\eta_{\delta}\right\|_{L_{1}\left(\mathbb{R}^{n}\right)}=1$ и $\left\|g_{\delta}\right\|_{L_{1}\left(\mathbb{R}^{n}\right)}=1$.

Так как $D_{1} \eta_{\delta}=\delta^{-1}\left(D_{1} \eta\right)_{\delta}$, то из (7) получаем неравенство

$$
\left\|D^{\alpha} \omega\right\|_{L_{\infty}\left(\mathbb{R}^{n}\right)} \leqslant 2^{n} v_{n}^{-1}\left(4\left\|D_{1} \eta\right\|_{L_{1}\left(B_{1}\right)}\right)^{|\alpha|} l^{|\alpha|} .
$$

Используя вид функции $\eta$ и переходя к полярным координатам, получим оценку

$$
\left\|D_{1} \eta\right\|_{L_{1}\left(B_{1}\right)}=(n+1) v_{n}^{-1} \int_{|x| \leqslant 1} \frac{\left|x_{1}\right|}{|x|} d x \leqslant(n+1) \leqslant 2^{n} .
$$

Последнее доказывает п. а) теоремы $1\left(C_{1}=8\right)$.

ЗАмЕчаниЕ 1 . Подобное теореме 1 утверждение можно сформулировать и о функциях типа "шапочки". 
Teорема $1^{\prime}$. а) Существует число $C_{3}>0$ и для любых $\delta>0, \quad n, l \in \mathbb{N} u$ любого множества $G \subset \mathbb{R}^{n}$ существует функция $\mu \in C^{\infty}\left(\mathbb{R}^{n}\right)$, удовлетворяющие следующим условиям:

1) $\operatorname{supp} \mu \subset G^{\delta}$;

2) $\mu(x)=1, \quad x \in G$;

3) $0 \leqslant \mu(x) \leqslant 1, \quad x \in \mathbb{R}^{n}$;

4) $\forall \alpha \in \mathbb{N}_{0}^{n}:|\alpha| \leqslant l$

$$
\left\|D^{\alpha} \mu\right\|_{L_{\infty}\left(\mathbb{R}^{n}\right)} \leqslant C_{3}^{n|\alpha|} \delta^{-|\alpha|} l^{|\alpha|} .
$$

б) При выполнении условий 1)-3) в оценке, приведенной в 4), показатель степени l также нельзя заменить на $\sigma|\alpha|$, где $\sigma<1$.

ДокАЗАтЕльство. Докажем п. а). П. б) доказывается в конще $\S 7$.

Пусть $\chi_{G} \frac{\delta}{2}-$ характеристическая функция множества $G^{\frac{\delta}{2}}$. Взяв вместо $\chi_{1}$ в доказательстве теоремы 1 функцию $\chi_{G} \frac{\delta}{2}$, построим функцию

$$
\mu=\left(A_{\theta}^{l} \chi_{G} \frac{\delta}{2}\right) * g_{\delta}, \quad \theta=\frac{\delta}{2(l+1)} .
$$

Условия 1)-3) следуют из свойств оператора усреднения.

Применяя неравенства (2), (3), получаем условие 4$)$ с $C_{3}=C_{1}$. Теорема $1^{\prime}$ доказана.

ЗАМЕЧАНИЕ 2. Если условие 1) теоремы 1 заменить на условие

$\left.1^{\prime}\right) \operatorname{supp} \omega \subset\left\{x \in \mathbb{R}^{n}:\left|x_{i}\right| \leqslant 1, i=1, \ldots, n\right\}$,

то справедливо утверждение, аналогичное теореме 1 с оценкой производных, не зависяшей от $n$.

TeOpema $1^{\prime \prime}$. а) Cуществует число $C_{4}>0$ и для любьх $n, l \in \mathbb{N}$ существует функиия $\omega \in C_{0}^{\infty}\left(\mathbb{R}^{n}\right)$, удовлетворяющие условию $\left.1^{\prime}\right)$, условиям 2), 3) из теоремы 1 и условию

$\left.4^{\prime \prime}\right) \quad \forall \alpha \in \mathbb{N}_{0}^{n}:|\alpha| \leqslant l$

$$
\left\|D^{\alpha} \omega\right\|_{L_{\infty}\left(\mathbb{R}^{n}\right)} \leqslant C_{4}^{|\alpha|} l^{|\alpha|}
$$

б) Оценка в $4^{\prime \prime}$ ) неулучшаема в смысле, определенном в теореме 1, б).

ДокАЗАТЕЛЬСТво. Приведем доказательство п. а); п. б) будет доказан в конце $\S 7$.

Положим $\omega(x)=\prod_{i=1}^{n} \widetilde{\omega}\left(x_{i}\right)$, где $\widetilde{\omega}-$ определенная в теореме 1 функция для $n=1$. Из п. 4) теоремы 1 следуют условия $\left.\left.\left.\left.1^{\prime}\right), 2\right), 3\right), 4^{\prime \prime}\right)$ с константой $C_{4}=8$. 


\section{2. Ядра усреднения, ортогональные полиномам.}

Teорема 2. а) Существует иисло $C_{5}>0$ и для любых $n, l \in \mathbb{N}$ cуществует функиия $\omega \in C_{0}^{\infty}\left(\mathbb{R}^{n}\right)$, удовлетворяющие следующим условиям:

1) $\operatorname{supp} \omega \subset\left\{x \in \mathbb{R}^{n}: \frac{1}{2}<x_{i}<1, i=1, \ldots, n\right\}$;

2) $\int_{\mathbb{R}^{n}} \omega(x) d x=1$

3) $\forall \alpha \in N_{0}^{n}:|\alpha| \leqslant l$

$$
\int_{\mathbb{R}^{n}} \omega(x) x^{\alpha} d x=0
$$

əде $x^{\alpha}=x_{1}^{\alpha_{1}} \ldots x_{n}^{\alpha_{n}}$

4) $\forall \alpha \in \mathbb{N}_{0}^{n}: \quad|\alpha| \leqslant l$

$$
\left\|D^{\alpha} \omega\right\|_{L_{\infty}\left(\mathbb{R}^{n}\right)} \leqslant C_{5}^{l n} l^{|\alpha|}
$$

ДоКАЗАТЕЛЬСТВО. Заметим, что если построена функция $\psi$ одной переменной со свойствами 1)-4), то функцию $\omega$ можно определить следующим образом:

$$
\omega(x)=\prod_{i=1}^{n} \psi\left(x_{i}\right)
$$

Пусть $\widehat{\omega}-$ функция из теоремы 1 для $n=1$. Рассмотрим функцию $\widetilde{\omega}$, определяемую равенством

$$
\widetilde{\omega}(x)=\frac{8}{l+1} \widehat{\omega}\left(\frac{8 x-14 l-6}{l+1}\right) .
$$

Из теоремы 1 для $n=1$ вытекают следуюшие свойства функции $\widetilde{\omega}$ :

$\left.1^{\prime}\right) \operatorname{supp} \widetilde{\omega} \subset\left(\frac{3 l+1}{2}, 2 l+1\right)$;

$\left.2^{\prime}\right) \int_{\mathbb{R}^{1}} \widetilde{\omega}(x) d x=1$

$\left.3^{\prime}\right) \forall k \in \mathbb{N}_{0}, k \leqslant l$

$$
\left\|\widetilde{\omega}^{(k)}\right\|_{L_{\infty}\left(\mathbb{R}^{1}\right)} \leqslant 8^{k} l^{k}\left(\frac{8}{l+1}\right)^{k+1} \leqslant 4(64)^{k} .
$$

Положим $\psi(x)=\sum_{k=1}^{l+1} a_{k} \widetilde{\omega}\left(b_{k} x\right), x \in \mathbb{R}^{1}$, где $b_{k}=2 l+k$, а коэффициенты $a_{k}$ определяются как решения системы уравнений

$$
\left\{\begin{array}{l}
\sum_{k=1}^{l+1} \frac{a_{k}}{b_{k}}=1, \\
\sum_{k=1}^{l+1} \frac{a_{k}}{b_{k}^{m+1}}=0, \quad m=1, \ldots, l .
\end{array}\right.
$$

Тогда

$$
\begin{aligned}
\int_{-\infty}^{+\infty} \psi(x) d x & =\sum_{k=1}^{l+1} a_{k} \int_{-\infty}^{+\infty} \widetilde{\omega}\left(b_{k} x\right) d x=1 \\
\int_{-\infty}^{+\infty} \psi(x) x^{m} d x & =\sum_{k=1}^{l+1} a_{k} \int_{-\infty}^{+\infty} \widetilde{\omega}\left(b_{k} x\right) x^{m} d x=0, \quad m=1, \ldots, l
\end{aligned}
$$


Определитель системы, определяющей $a_{k}$, сводится к определителю Вандермонда и имеет вид

$$
\Delta=\prod_{q=1}^{l+1} b_{q}^{-1} \prod_{1 \leqslant j<i \leqslant l+1}\left(b_{i}^{-1}-b_{j}^{-1}\right)
$$

Согласно правилу Крамера $a_{k}=\frac{\Delta_{k}}{\Delta}$, где определитель $\Delta_{k}$ после разложения по $k$-у столбцу также сводится к определителю Вандермонда:

$$
\Delta_{k}=(-1)^{k+1} \prod_{\substack{q=1 \\ q \neq k}}^{l+1} b_{q}^{-2} \prod_{\substack{1 \leqslant j<i \leqslant l+1 \\ i \neq k, j \neq k}}\left(b_{i}^{-1}-b_{j}^{-1}\right)
$$

Следовательно, $\left|a_{k}\right|=\left|b_{k}^{l+1} \prod_{\substack{i \neq 1 \\ i \neq k}}^{l+1}\left(b_{k}-b_{i}\right)^{-1}\right|$.

Подставляя значение $b_{k}$ в последнюю формулу, получаем оценку

$$
\left|a_{k}\right| \leqslant \frac{(4 l)^{l+1}}{(k-1) !(l-k+1) !} \leqslant(8 e)^{l} \frac{l !}{(k-1) !(l-k+1) !} .
$$

Отсюда следует неравенство

$$
\left|a_{k}\right| \leqslant(16 e)^{l}
$$

Так как $\psi^{(m)}(x)=\sum_{k=1}^{l+1} b_{k}^{m} a_{k} \widetilde{\omega}^{(m)}\left(b_{k} x\right)$, то при $m \leqslant l$ из (13) и свойства $\left.3^{\prime}\right)$ получим оценку производной

$$
\left|\psi^{(m)}(x)\right| \leqslant \sum_{k=1}^{l+1}(4 l)^{m}(16 e)^{l} 4(64)^{m} \leqslant l^{m}\left(2^{15} e\right)^{l}
$$

т.е. при $n=1$ справедливо неравенство (11) с $C_{5}=2^{15} e$.

Далее, согласно $1^{\prime}$ )

$$
\operatorname{supp} \psi \subset \bigcup_{k=1}^{l+1} \operatorname{supp} \widetilde{\omega}\left(b_{k} x\right) \subset \bigcup_{k=1}^{l+1}\left(\frac{3 l+1}{2 b_{k}}, \frac{2 l+1}{b_{k}}\right) \subset\left(\frac{1}{2}, 1\right)
$$

Теорема 2 доказана.

\section{§ 2. Интегральное представление}

Пусть $-\infty<a_{j}<b_{j}<+\infty, j=1, \ldots, n, \Pi_{i}=\left\{x \in \mathbb{R}^{i}: a_{j}<x_{j}<b_{j}\right.$, $j=1, \ldots, i\}, \Pi \equiv \Pi_{n}$.

ЛЕмма 1. Для любой функиии $f \in C(\bar{\Pi}), D_{1}^{l} f \in C(\bar{\Pi}), \ldots, D_{n}^{l} f \in C(\bar{\Pi}), \quad l \in$ $\mathbb{N}$, существуют многочлен $P \in L_{\infty}(\Pi \times \Pi)$ степени не выше $l-1$ по каждой 
переменной и функиии $Q_{i} \in L_{\infty}\left(\Pi_{i} \times \Pi_{i}\right), \quad i=1, \ldots, n$, удовлетворяющие условиям

$$
\begin{aligned}
& \left|P\left(x_{1}, \ldots, x_{n} ; \xi_{1}, \ldots, \xi_{n}\right)\right| \leqslant C_{1}^{l n} \prod_{j=1}^{n}\left(b_{j}-a_{j}\right)^{-1} \\
& \left|Q_{i}\left(x_{1}, \ldots, x_{i} ; \xi_{1}, \ldots, \xi_{i}\right)\right| \leqslant C_{1}^{l n} l^{-l}\left(\prod_{j=1}^{i-1}\left(b_{j}-a_{j}\right)^{-1}\right)\left(b_{i}-a_{i}\right)^{l-1}
\end{aligned}
$$

c некоторой абсолютной постоянной $C_{1}>0$, такие, что имеет место представление

$$
\begin{gathered}
f(x)=\int_{\Pi} P(x, \xi) f(\xi) d \xi+\sum_{i=1}^{n} \int_{\Pi_{i}} Q_{i}\left(x_{1}, \ldots, x_{i} ; \xi_{1}, \ldots, \xi_{i}\right) \\
\times\left(D_{i}^{l} f\right)\left(\xi_{1}, \ldots, \xi_{i}, x_{i+1}, \ldots, x_{n}\right) d \xi_{1} \ldots d \xi_{i} .
\end{gathered}
$$

ДокаЗАТЕЛЬСТво. Рассмотрим случай $n=1$. Пусть $-\infty<a<b<+\infty$ и $f \in C^{l}([a, b])$; тогда согласно [6] функция $f$ при любом $x \in[a, b]$ представляется в виде

$$
f(x)=\int_{a}^{b} p(x, \xi) f(\xi) d \xi+\int_{a}^{b} q(x, \xi) f^{(l)}(\xi) d \xi
$$

где

$$
\begin{aligned}
p(x, \xi) & =\sum_{m=0}^{l-1} \alpha_{m}(x-\xi)^{m} \lambda^{(m)}(\xi), \\
\alpha_{m} & =\frac{1}{m !} \sum_{j=m}^{l-1}(-1)^{j} C_{j}^{m}, \\
q(x, \xi) & =\frac{(x-\xi)^{l-1}}{(l-1) !} \Lambda(x, \xi),
\end{aligned}
$$

а измеримая функция $\Lambda$ такова, что $|\Lambda(x, \xi)| \leqslant 1$.

Функция $\lambda$ имеет вид

$$
\lambda(\xi)=\frac{1}{b-a} \omega\left(\frac{\xi-a}{b-a}\right),
$$

где $\omega \in C_{0}^{\infty}(0,1)$ и $\int_{0}^{1} \omega(x) d x=1$.

Выбрав в качестве $\omega$ функцию, удовлетворяющую условиям теоремы 1 , получим, что существует такое число $C_{2} \geqslant 1$, что при любых $m \leqslant l$ для любых $\xi \in[a, b]$

$$
\left|\lambda^{(m)}(\xi)\right| \leqslant C_{2}^{m} l^{m}(b-a)^{-m-1} .
$$

Из (5)-(8) вытекают следуюшие оценки: для любых $x, \xi \in[a, b]$

$$
|p(x, \xi)| \leqslant C_{3}^{l}(b-a)^{-1}
$$


где $C_{3}=2 e C_{2}$ (достаточно воспользоваться тем, что $\left|\alpha_{m}\right| \leqslant \frac{2^{l}}{m !}$ и $\left.\sum_{m=0}^{l-1} \frac{l^{m}}{m !} \leqslant e^{l}\right)$, и

$$
|q(x, \xi)| \leqslant \frac{(b-a)^{l-1}}{(l-1) !} \leqslant e^{2 l} l^{-l}(b-a)^{l-1}
$$

(мы учли, что $\left.\frac{l^{l}}{(l-1) !} \leqslant \frac{(2 l)^{l-1}}{(l-1) !} \leqslant e^{2 l}\right)$.

Согласно [6] представление (3) имеет место для функций $P$ и $Q_{i}$ вида

$$
\begin{aligned}
P(x, \xi) & =p_{1}\left(x_{1}, \xi_{1}\right) \ldots p_{n}\left(x_{n}, \xi_{n}\right) \\
Q_{i}(x, \xi) & =p_{1}\left(x_{1}, \xi_{1}\right) \ldots p_{i-1}\left(x_{i-1}, \xi_{i-1}\right) q_{i}\left(x_{i}, \xi_{i}\right)
\end{aligned}
$$

где при определении функций $p_{j}$ и $q_{j}$ вместо функции $\lambda$ рассматривается функция

$$
\lambda_{j}(\xi)=\frac{1}{b_{j}-a_{j}} \omega\left(\frac{\xi-a_{j}}{b_{j}-a_{j}}\right)
$$

Отсюда согласно (9), (10) следует доказательство леммы $1\left(\right.$ с $\left.C_{1}=C_{3} e^{2}\right)$.

ЗАмЕчАниЕ. В одномерном случае наряду с (9) и (10) нам понадобится еше следуюшая оценка для производных многочлена $p(x, \xi)$ : сушествует такое число $C_{4}>0$, что при $0 \leqslant s \leqslant l-1$ для любых $-\infty<x, \xi<+\infty$

$$
\left|\frac{\partial^{s} p}{\partial x^{s}}(x, \xi)\right| \leqslant C_{4}^{l} l^{s}\left(1+|x-\xi|^{l-1-s}\right)\left(1+(b-a)^{-l+1}\right)(b-a)^{-1}
$$

(при $s=0$ для $x, \xi \in[a, b]$ из (11) следует (9)).

Действительно,

$$
\begin{aligned}
\left|\frac{\partial^{s} p}{\partial x^{s}}(x, \xi)\right| & =\left|\sum_{m=s}^{l-1} \frac{\alpha_{m} m !}{(m-s) !}(x-\xi)^{m-s} \lambda^{(m)}(\xi)\right| \\
& \leqslant 2^{l} \sum_{m=s}^{l-1} \frac{\left(C_{2} l\right)^{m}}{(m-s) !}|x-\xi|^{m-s}(b-a)^{-m-1} \\
& \leqslant 2^{l}\left(1+|x-\xi|^{l-1-s}\right)\left(1+(b-a)^{-l+1}\right)(b-a)^{-1} \sum_{m=s}^{l-1} \frac{\left(C_{2} l\right)^{m}}{(m-s) !} \\
& \leqslant 2^{l} e^{C_{2} l}\left(C_{2} l\right)^{s}\left(1+|x-\xi|^{l-1-s}\right)\left(1+(b-a)^{-l+1}\right)(b-a)^{-1},
\end{aligned}
$$

откуда и следует (11) (с $\left.C_{4}=2 C_{2} e^{C_{2}}\right)$.

§ 3. Двусторонняя оценка минимальной нормы операторов проддлжения для пространств Соболева в одномерном случае

\section{1. Оценка сверху.}

ЛЕмма 2. Для любых $l \in \mathbb{N}$ существует такой оператор продолжсения $T$, что для любих $1 \leqslant p \leqslant+\infty \quad T: W_{p}^{l}(-\infty, 0) \rightarrow W_{p}^{l}(-\infty,+\infty)$, причем

$$
\|T\|_{W_{p}^{l}(-\infty, 0) \rightarrow W_{p}^{l}(-\infty,+\infty)} \leqslant 8^{l} .
$$


ДокАЗАТЕЛЬСТво. Определим оператор продолжения по схеме Хестенса следуюшим образом: для любой функции $f \in W_{p}^{l}(-\infty, 0)$

$$
(T f)(x)= \begin{cases}f(x), & x \in(-\infty, 0), \\ \sum_{k=1}^{l} \alpha_{k} f\left(-\beta_{k} x\right), & x \in(0,+\infty),\end{cases}
$$

где $\beta_{k}>0$ и

$$
\sum_{k=1}^{l} \alpha_{k}\left(-\beta_{k}\right)^{s}=1, \quad s=0, \ldots, l-1 .
$$

Решение системы (3) записывается в виде

$$
\alpha_{k}=(-1)^{l-1} \prod_{\substack{j=1 \\ j \neq k}}^{l}\left(1+\beta_{j}\right)\left(\beta_{k}-\beta_{j}\right)^{-1}
$$

Положим

$$
\beta_{k}=\frac{k}{l}, \quad k=1, \ldots, l
$$

тогда

$$
\alpha_{k}=\frac{(-1)^{k-1} k}{l+k} C_{2 l}^{l} C_{l}^{k}, \quad k=1, \ldots, l
$$

Из (5) следует, что

$$
\left|\alpha_{k}\right| \leqslant \frac{k}{l} 4^{l} C_{l}^{k}
$$

Согласно (4) и (6)

$$
\begin{aligned}
\|T f\|_{W_{p}^{l}(0,+\infty)} & =\|T f\|_{L_{p}(0,+\infty)}+\left\|(T f)^{(l)}\right\|_{L_{p}(0,+\infty)} \\
& \leqslant\left(\sum_{k=1}^{l}\left|\alpha_{k}\right| \beta_{k}^{-\frac{1}{p}}\right)\|f\|_{L_{p}(-\infty, 0)}+\left(\sum_{k=1}^{l}\left|\alpha_{k}\right| \beta_{k}^{l-\frac{1}{p}}\right)\left\|f^{(l)}\right\|_{L_{p}(-\infty, 0)} \\
& \leqslant\left(\sum_{k=1}^{l}\left|\alpha_{k}\right| \beta_{k}^{-\frac{1}{p}}\right)\|f\|_{W_{p}^{l}(-\infty, 0)} \\
& \leqslant 4^{l} \sum_{k=1}^{l}\left(\frac{k}{l}\right)^{1-\frac{1}{p}} C_{l}^{k}\|f\|_{W_{p}^{l}(-\infty, 0)} \leqslant\left(8^{l}-1\right)\|f\|_{W_{p}^{l}(-\infty, 0)} .
\end{aligned}
$$

Обозначим через $f^{(k)}(-0)$ и соответственно через $(T f)^{(k)}(+0)$ граничные значения в точке 0 производных функций $f \in W_{p}^{l}(-\infty, 0)$ и $T f \in W_{p}^{l}(0,+\infty)$ (при $k \leqslant l-1$ они существуют).

Согласно условию (3)

$$
(T f)^{(k)}(+0)=f^{(k)}(-0), \quad k=0, \ldots, l-1,
$$

поэтому $T f \in W_{p}^{l}(-\infty,+\infty)$. Кроме того,

$$
\|T f\|_{W_{p}^{l}(-\infty,+\infty)} \leqslant\|f\|_{W_{p}^{l}(-\infty, 0)}+\|T f\|_{W_{p}^{l}(0,+\infty)} \leqslant 8^{l}\|f\|_{W_{p}^{l}(-\infty, 0)},
$$

что и требовалось доказать. 
СлеДСТВИЕ. Для любых $l \in \mathbb{N},-\infty<a<b<+\infty$ существует такой оператор продолжения $T$, что для любы $1 \leqslant p \leqslant+\infty \quad T: W_{p}^{l}(a, b) \rightarrow W_{p}^{l}(2 a-b$, $2 b-a)$, причем

$$
\|T\|_{W_{p}^{l}(a, b) \rightarrow W_{p}^{l}(2 a-b, 2 b-a)} \leqslant 2 \cdot 8^{l} .
$$

ДоКАЗАТЕЛЬСТво. Определим оператор продолжения следующим образом: для любой функции $f \in W_{p}^{l}(a, b)$

$$
(T f)(x)= \begin{cases}\sum_{k=1}^{l} \alpha_{k} f\left(a+\beta_{k}(a-x)\right), & x \in(2 a-b, a), \\ f(x), & x \in(a, b), \\ \sum_{k=1}^{l} \alpha_{k} f\left(b+\beta_{k}(b-x)\right), & x \in(b, 2 b-a) .\end{cases}
$$

После оценок, аналогичных проведенным в доказательстве леммы 2 , приходим к неравенству

$$
\|T f\|_{W_{p}^{l}(2 a-b, 2 b-a)} \leqslant\left(1+2 \sum_{k=1}^{l}\left|\alpha_{k}\right| \beta_{k}^{-\frac{1}{p}}\right)\|f\|_{W_{p}^{l}(a, b)},
$$

откуда и следует неравенство (7).

ЗАмЕчАниЕ. Из приведенных доказательств следует, что наряду с неравенством (7) выполняются также неравенства

$$
\|T\|_{L_{p}(a, b) \rightarrow L_{p}(2 a-b, 2 a+b)} \leqslant 2 \cdot 8^{l}
$$

и

$$
\|T\|_{w_{p}(a, b) \rightarrow w_{p}(2 a-b, 2 a+b)} \leqslant 2 \cdot 8^{l} .
$$

Лемма 3. Существует число $C_{1}>0$ и для любих $l \in \mathbb{N},-\infty \leqslant a<b \leqslant+\infty$ существует такой оператор продолжения $T$, что для любых $1 \leqslant p \leqslant+\infty$ $T: W_{p}^{l}(a, b) \rightarrow W_{p}^{l}(-\infty,+\infty)$, причем

$$
\|T\|_{W_{p}^{l}(a, b) \rightarrow W_{p}^{l}(-\infty,+\infty)} \leqslant C_{1}^{l}\left(1+\frac{l^{l}}{(b-a)^{l}}\right) .
$$

ДокАЗАТЕльСтво. Если $b-a=\infty$, то неравенство (11) следует из неравенства (1). Пусть теперь $-\infty<a<b<+\infty$. Рассмотрим оператор $T$, определяемый равенством (8), и доопределим $T f$ вне интервала $(2 a-b, 2 b-a)$ нулем. Обозначим через $\varphi$ функцию типа "шапочки”, удовлетворяющую условиям теоремы 1 ' для $G=(a, b)$ и $\delta=b-a$; тогда, в частности, для некоторого $C_{2}>0$ для любых $k \in \mathbb{N}_{0}: k \leqslant l$

$$
\left\|\varphi^{(k)}\right\|_{L_{\infty}(-\infty, \infty)} \leqslant\left(\frac{C_{2} l}{b-a}\right)^{k} .
$$

Определим оператор продолжения $T_{1}$ равенством

$$
\left(T_{1} f\right)(x)=(T f)(x) \varphi(x), \quad x \in(-\infty,+\infty) .
$$


Прежде всего, согласно (9)

$$
\left\|T_{1} f\right\|_{L_{p}(-\infty,+\infty)} \leqslant 2 \cdot 8^{l}\|f\|_{L_{p}(a, b)} .
$$

Для оценок производных $\left(T_{1} f\right)^{(l)}$ нам понадобится следующее неравенство для норм промежуточных производных [8]:

$$
\begin{aligned}
\left\|g^{(k)}\right\|_{L_{p}(a, b)} & \leqslant C_{3}^{l}\left(\left(\frac{k}{b-a}\right)^{k}\|g\|_{L_{p}(a, b)}+\left(\frac{b-a}{l-k}\right)^{l-k}\left\|g^{(l)}\right\|_{L_{p}(a, b)}\right) \\
& \leqslant C_{3}^{l}\left(\left(\frac{k}{b-a}\right)^{k}+\left(\frac{b-a}{l-k}\right)^{l-k}\right)\|g\|_{W_{p}^{l}(a, b)},
\end{aligned}
$$

где $C_{3}>0$ - абсолютная постоянная.

Из (12), (15) и (7) следует, что

$$
\begin{aligned}
& \left\|\left(T_{1} f\right)^{(l)}\right\|_{L_{p}(-\infty,+\infty)} \leqslant \sum_{k=0}^{l} C_{l}^{k}\left\|\varphi^{(l-k)}\right\|_{L_{\infty}(-\infty,+\infty)}\left\|(T f)^{(k)}\right\|_{L_{p}(2 a-b, 2 b-a)} \\
& \leqslant C_{3}^{l}\left(\sum_{k=0}^{l} C_{l}^{k}\left(\frac{C_{2} l}{b-a}\right)^{l-k}\left[\left(\frac{k}{3(b-a)}\right)^{k}+\left(\frac{3(b-a)}{l-k}\right)^{l-k}\right]\right)\|T f\|_{W_{p}^{l}(2 a-b, 2 b-a)} \\
& \leqslant C_{3}^{l}\left[\left(\sum_{k=0}^{l} C_{l}^{k} 3^{-k} C_{2}^{l-k}\right) \frac{l^{l}}{(b-a)^{l}}+\sum_{k=0}^{l} C_{l}^{k} e^{k}\left(3 C_{2}\right)^{l-k}\right]\|T f\|_{W_{p}^{l}(2 a-b, 2 b-a)} \\
& \leqslant 2\left(8 C_{3}\right)^{l}\left(\left(C_{2}+\frac{1}{3}\right)^{l} \frac{l^{l}}{(b-a)^{l}}+\left(e+3 C_{2}\right)^{l}\right)\|f\|_{W_{p}^{l}(a, b)} \\
& \quad \leqslant C_{4}^{l}\left(1+\frac{l^{l}}{(b-a)^{l}}\right)\|f\|_{W_{p}^{l}(a, b)},
\end{aligned}
$$

где $C_{4}=16 C_{3}\left(e+3 C_{2}\right)$. Из этого неравенства и неравенства (14) следует, что

$$
\left\|T_{1} f\right\|_{W_{p}^{l}(-\infty,+\infty)} \leqslant C_{1}^{l}\left(1+\frac{l^{l}}{(b-a)^{l}}\right)\|f\|_{W_{p}^{l}(a, b)}
$$

$\left(\right.$ с $\left.C_{1}=2 C_{4}\right)$, что и требовалось доказать.

Лемма 4. Существует число $C_{5}>0$ и для любых $\varepsilon \in(0,1), \quad l \in \mathbb{N} c y$ ществует такой оператор продолжения $T_{\varepsilon}$, что для любых $1 \leqslant p \leqslant+\infty$ $T_{\varepsilon}: W_{p}^{l}(0, \varepsilon) \rightarrow W_{p}^{l}(-\infty,+\infty)$, причем

$$
\left\|T_{\varepsilon}\right\|_{W_{p}^{l}(0, \varepsilon) \rightarrow W_{p}^{l}(-\infty,+\infty)} \leqslant C_{5}^{l} l^{l} \varepsilon^{-l+\frac{1}{p^{\prime}}}
$$


ДокаЗАТЕльСтво. 1 . Пусть $f \in W_{p}^{l}(0, \varepsilon)$. Применяя интегральное представление (4) из $\S 2$ к функции $f(\varepsilon x)$, получим, что для любого $x \in(0,1)$

$$
f(\varepsilon x)=\int_{0}^{1} p(x, \xi) f(\varepsilon \xi) d \xi+\int_{0}^{1} q(x, \xi)(f(\varepsilon \xi))_{\xi}^{(l)} d \xi .
$$

Заменяя $\varepsilon x$ на $x$, получим следуюшее интегральное представление для функций $f \in W_{p}^{l}(0, \varepsilon)$ : для любых $x \in(0, \varepsilon)$

$$
f(x)=\int_{0}^{1} p\left(\frac{x}{\varepsilon}, \xi\right) f(\varepsilon \xi) d \xi+\varepsilon^{l} \int_{0}^{1} q\left(\frac{x}{\varepsilon}, \xi\right) f^{(l)}(\varepsilon \xi) d \xi \equiv\left(P_{\varepsilon} f\right)(x)+\left(Q_{\varepsilon} f\right)(x) .
$$

Так как $p(x, \xi)$ - многочлен степени не вьше $l-1$ по $x$, то отсюда следует, что для любых $x \in(0, \varepsilon)$

$$
\left(Q_{\varepsilon} f\right)^{(l)}(x)=f^{(l)}(x) .
$$

2. Докажем, что существует такое $C_{6}>0$, что для любых $s \in \mathbb{N}_{0}: 0 \leqslant s \leqslant l-1$

$$
\left\|\left(P_{\varepsilon} f\right)^{(s)}\right\|_{L_{p}(-2,2)} \leqslant C_{6}^{l} l^{s} \varepsilon^{-l+\frac{1}{p^{\prime}}}\|f\|_{L_{p}(0, \varepsilon)}
$$

и

$$
\left\|Q_{\varepsilon} f\right\|_{L_{p}(0, \varepsilon)} \leqslant C_{6}^{l} l^{-l} \varepsilon^{l}\left\|f^{(l)}\right\|_{L_{p}(0, \varepsilon)} .
$$

Для получения оценки (19) воспользуемся тем, что для $|x| \leqslant 2$ и $0 \leqslant \xi \leqslant 1$ $\left|\frac{x}{\varepsilon}-\xi\right| \leqslant \frac{3}{\varepsilon}$, и согласно неравенству (11) из $\S 2$

$$
\left\|p^{(s)}\left(\frac{x}{\varepsilon}, \xi\right)\right\|_{L_{p, x}(-2,2)} \leqslant 4^{\frac{1}{p}}\left\|p^{(s)}\left(\frac{x}{\varepsilon}, \xi\right)\right\|_{L_{\infty, x}(-2,2)} \leqslant C_{7}^{l} l^{s} \varepsilon^{-l+1+s},
$$

где $C_{7}=48 C_{4}$ (здесь $C_{4}$ - постоянная из неравенства (11) из $\S 2$, в котором $a=0$, $\left.b=1 ; p^{(s)}(x, \xi)=\frac{\partial^{s} p}{\partial x^{s}}(x, \xi)\right)$. Согласно неравенству Минковского отсюда следует, что

$$
\begin{aligned}
\left\|\left(P_{\varepsilon} f\right)^{(s)}\right\|_{L_{p}(-2,2)} & =\left\|\varepsilon^{-s} \int_{0}^{1} p^{(s)}\left(\frac{x}{\varepsilon}, \xi\right) f(\varepsilon \xi) d \xi\right\|_{L_{p}(-2,2)} \\
& \leqslant \varepsilon^{-s} \int_{0}^{1}\left\|p^{(s)}\left(\frac{x}{\varepsilon}, \xi\right)\right\|_{L_{p, x}(-2,2)}|f(\varepsilon \xi)| d \xi \\
& \leqslant C_{7}^{l} l^{s} \varepsilon^{-l+1} \int_{0}^{1}|f(\varepsilon \xi)| d \xi \leqslant C_{7}^{l} l^{s} \varepsilon^{-l+1}\|f(\varepsilon \xi)\|_{L_{p, \xi}(0,1)} \\
& =C_{7}^{l} l^{s} \varepsilon^{-l+1-\frac{1}{p}}\|f\|_{L_{p}(0, \varepsilon)},
\end{aligned}
$$

и мы получили (19).

Для получения неравенства (20) достаточно заметить, что согласно неравенству (10) из $\S 2\left(\right.$ с $a=0, b=1 ;$ при этом $\left.\frac{x}{\varepsilon}, \xi \in[a, b]\right)$

$$
\begin{aligned}
\left\|Q_{\varepsilon} f\right\|_{L_{p}(0, \varepsilon)} & =\varepsilon^{l}\left\|\int_{0}^{1} q\left(\frac{x}{\varepsilon}, \xi\right) f^{(l)}(\varepsilon \xi) d \xi\right\|_{L_{p, x}(0, \varepsilon)} \leqslant e^{2 l} l^{-l} \varepsilon^{l+\frac{1}{p}} \int_{0}^{1}\left|f^{(l)}(\varepsilon \xi)\right| d \xi \\
& \leqslant e^{2 l} l^{-l} \varepsilon^{l+\frac{1}{p}}\left\|f^{(l)}(\varepsilon \xi)\right\|_{L_{p}(0,1)}=e^{2 l} l^{-l} \varepsilon^{l}\left\|f^{(l)}\right\|_{L_{p}(0, \varepsilon)} .
\end{aligned}
$$


3. Построим теперь оператор продолжения $T_{\varepsilon}: W_{p}^{l}(0, \varepsilon) \rightarrow W_{p}^{l}(-\infty,+\infty)$. Пусть $T$ - оператор продолжения из леммы 3 , т.е. $T: W_{p}^{l}(0,1) \rightarrow W_{p}^{l}(-\infty,+\infty)$. Обозначим через $\varphi$ функцию типа "шапочки", удовлетворяющую условиям теоремы 1 ' для $G=(0,1)$ и $\delta=1$; тогда, в частности, для некоторого $C_{8}>0$ для любых $k \in \mathbb{N}: k \leqslant l$

$$
\left\|\varphi^{(k)}\right\|_{L_{\infty}(-\infty,+\infty)} \leqslant\left(C_{8} l\right)^{k} .
$$

Положим для любых $f \in W_{p}^{l}(0, \varepsilon)$

$$
T_{\varepsilon} f=\varphi\left(P_{\varepsilon} f\right)+\tau_{\varepsilon}^{-1} T\left(\tau_{\varepsilon} Q_{\varepsilon} f\right),
$$

где $\left(\tau_{\varepsilon} g\right)(x)=g(\varepsilon x)$ для $0<x<1$.

4. Используя неравенства (14) для $a=0, b=1$ (в наших обозначениях $T_{1} \equiv T$ ), неравенство (19) для $s=0$ и неравенство (20), получим, что

$$
\begin{aligned}
& \left\|T_{\varepsilon} f\right\|_{L_{p}(-\infty,+\infty)} \leqslant\left\|\varphi\left(P_{\varepsilon} f\right)\right\|_{L_{p}(-\infty,+\infty)}+\left\|\tau_{\varepsilon}^{-1} T\left(\tau_{\varepsilon} Q_{\varepsilon} f\right)\right\|_{L_{p}(-\infty,+\infty)} \\
& \leqslant\left\|P_{\varepsilon} f\right\|_{L_{p}(-2,2)}+\varepsilon^{\frac{1}{p}}\left\|T\left(\tau_{\varepsilon} Q_{\varepsilon} f\right)\right\|_{L_{p}(-\infty,+\infty)} \\
& \leqslant C_{6}^{l} \varepsilon^{-l+\frac{1}{p^{\prime}}}\|f\|_{L_{p}(0, \varepsilon)}+16^{l} \varepsilon^{\frac{1}{p}}\left\|\tau_{\varepsilon} Q_{\varepsilon} f\right\|_{L_{p}(0,1)} \\
& =C_{6}^{l} \varepsilon^{-l+\frac{1}{p^{\prime}}}\|f\|_{L_{p}(0, \varepsilon)}+16^{l}\left\|Q_{\varepsilon} f\right\|_{L_{p}(0, \varepsilon)} \\
& \leqslant C_{6}^{l} \varepsilon^{-l+\frac{1}{p^{\prime}}}\|f\|_{L_{p}(0, \varepsilon)}+\left(16 C_{6}\right)^{l} l^{-l} \varepsilon^{l}\left\|f^{(l)}\right\|_{L_{p}(0, \varepsilon)}
\end{aligned}
$$

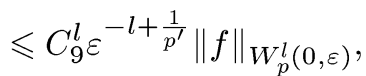

где $C_{9}=16 C_{6}$.

Далее,

$$
\left(T_{\varepsilon} f\right)^{(l)}=\sum_{k=0}^{l} C_{l}^{k} \varphi^{(k)}\left(P_{\varepsilon} f\right)^{(l-k)}+\varepsilon^{-l} \tau_{\varepsilon}^{-1}\left(T\left(\tau_{\varepsilon} Q_{\varepsilon} f\right)\right)^{(l)} .
$$

Откуда согласно неравенствам (11), (19), (20), (21) и равенству (18) следует, что

$$
\begin{aligned}
\left\|\left(T_{\varepsilon} f\right)^{(l)}\right\|_{L_{p}(-\infty,+\infty) \leqslant} \leqslant & \sum_{k=0}^{l} C_{l}^{k}\left(C_{8} l\right)^{k}\left\|\left(P_{\varepsilon} f\right)^{(l-k)}\right\|_{L_{p}(-2,2)} \\
& +\varepsilon^{-l+\frac{1}{p}}\left\|\left(T\left(\tau_{\varepsilon} Q_{\varepsilon} f\right)\right)^{(l)}\right\|_{L_{p}(-\infty,+\infty)} \\
\leqslant & \left(\sum_{k=0}^{l} C_{l}^{k}\left(C_{8} l\right)^{k} C_{6}^{l} l^{l-k}\right) \varepsilon^{-l+\frac{1}{p^{\prime}}}\|f\|_{L_{p}(0, \varepsilon)} \\
& +\left(2 C_{1}\right)^{l} l^{l} \varepsilon^{-l+\frac{1}{p^{\prime}}}\left(\left\|\tau_{\varepsilon} Q_{\varepsilon} f\right\|_{L_{p}(0,1)}+\left\|\left(\tau_{\varepsilon} Q_{\varepsilon} f\right)^{(l)}\right\|_{L_{p}(0,1)}\right) \\
= & l^{l}\left[\left(C_{6}\left(1+C_{8}\right)\right)^{l} \varepsilon^{-l+\frac{1}{p^{\prime}}}\|f\|_{L_{p}(0, \varepsilon)}\right. \\
& \left.+\left(2 C_{1}\right)^{l}\left(\varepsilon^{-l}\left\|Q_{\varepsilon}\right\|_{L_{p}(0, \varepsilon)}+\left\|f^{(l)}\right\|_{L_{p}(0, \varepsilon)}\right)\right] \\
\leqslant & l^{l}\left[\left(C_{6}\left(1+C_{8}\right)\right)^{l} \varepsilon^{-l+\frac{1}{p^{\prime}}}\|f\|_{L_{p}(0, \varepsilon)}\right. \\
& \left.+\left(2 C_{1}\right)^{l}\left(1+C_{6}\right)^{l}\left\|f^{(l)}\right\|_{L_{p}(0, \varepsilon)}\right] \leqslant C_{10}^{l} l \varepsilon^{l} \varepsilon^{-l+\frac{1}{p^{\prime}}}\|f\|_{W_{p}^{l}(0, \varepsilon)},
\end{aligned}
$$


где $C_{10}=\max \left\{C_{6}\left(1+C_{8}\right), 2 C_{1}\left(1+C_{6}\right)\right\}$.

Из полученных оценок следует искомое неравенство (16).

ЗАмЕчАниЕ. Для любых функций $f \in w_{p}^{l}(0, \varepsilon)$ рассмотрим оператор продолжения

$$
\widetilde{T}_{\varepsilon} f=P_{\varepsilon}(f)+\tau_{\varepsilon}^{-1} T\left(\tau_{\varepsilon} Q_{\varepsilon} f\right)
$$

Так как

$$
\left(\widetilde{T}_{\varepsilon} f\right)^{(l)}=\left(\tau_{\varepsilon}^{-1} T\left(\tau_{\varepsilon} Q_{\varepsilon} f\right)\right)^{(l)},
$$

то из проведенных вьше оценок следует, что

$$
\left\|\widetilde{T}_{\varepsilon}\right\|_{w_{p}^{l}(0, \varepsilon) \rightarrow w_{p}^{l}(-\infty,+\infty)} \leqslant C_{11}^{l} l^{l}
$$

где $C_{11}=2 C_{1}\left(1+C_{6}\right)$.

\section{2. Оценка снизу.}

ЛЕмма 5. Норма любого оператора продолжения $T: W_{p}^{l}(0, \varepsilon) \rightarrow$ $W_{p}^{l}(-\infty,+\infty)$ при любых $l \in \mathbb{N}, \quad 1 \leqslant p \leqslant \infty, \quad \varepsilon \in(0, \infty)$ удовлетворяет неравенству

$$
\|T\|_{W_{p}^{l}(0, \varepsilon) \rightarrow W_{p}^{l}(-\infty,+\infty)} \geqslant \frac{1}{3} 4^{l-1}(l-1) ! \varepsilon^{-l+\frac{1}{p^{\prime}}} .
$$

Мы приведем два доказательства леммы 5. Первое из них прямое: оно основано на подборе "наихудшей” для продолжения функции $f \in W_{p}^{l}(0, \varepsilon)$. Второе основано на приводимой ниже лемме 6 , в которой в самом обшем случае дается оценка снизу нормы любого оператора продолжения через наименьшие постоянные в неравенствах для норм промежуточных производных. В обоих доказательствах участвуют многочлены $Q_{l-1 ; p}$ степени $l-1$, наименее уклоняюшиеся от нуля в метрике $L_{p}(0,1)$.

ПЕРВОЕ ДОКАЗАТЕЛЬСТвО. Положим $f(x)=\frac{\varepsilon^{l-1}}{(l-1) !} Q_{l-1 ; p}\left(\frac{x}{\varepsilon}\right)$. Отметим, что $f^{(l-1)}(0)=1$. Из определения нормы оператора следует неравенство

$$
\begin{aligned}
\|T\| & \equiv\|T\|_{W_{p}^{l}(0, \varepsilon) \rightarrow W_{p}^{l}(-\infty,+\infty)} \geqslant \frac{\|T f\|_{W_{p}^{l}(-\infty,+\infty)}}{\|f\|_{L_{p}(0, \varepsilon)}} \\
& =\frac{(l-1) !}{\left\|Q_{l-1 ; p}\right\|_{L_{p}(0,1)}} \varepsilon^{-l+\frac{1}{p^{\prime}}}\|g\|_{W_{p}^{l}(-\infty,+\infty)}
\end{aligned}
$$

где $g=T f$

Так как $g \in W_{p}^{l}(-\infty,+\infty)$, то

$$
g^{(l-1)}(-0)=f^{(l-1)}(+0)=1,
$$

где через $g^{(l-1)}(-0)$ обозначено граничное значение производной порядка $l-1$ в точке 0 для функции $g$ (рассматриваемой как функция из $W_{p}^{l}(-\infty, 0)$ ). 
Из неравенства Колмогорова-Стейна следует, что

$$
\begin{aligned}
\left\|g^{(l-1)}\right\|_{L_{p}(-\infty,+\infty)} & \leqslant \frac{\pi}{2}\|g\|_{L_{p}(-\infty,+\infty)}^{\frac{1}{l}}\left\|g^{(l)}\right\|_{L_{p}(-\infty,+\infty)}^{1-\frac{1}{l}} \\
& \leqslant \frac{\pi}{2}\left(\frac{1}{l}\right)^{\frac{1}{l}}\left(1-\frac{1}{l}\right)^{1-\frac{1}{l}}\left(\|g\|_{L_{p}(-\infty,+\infty)}+\left\|g^{(l)}\right\|_{L_{p}(-\infty,+\infty)}\right) \\
& \leqslant \frac{\pi}{2}\|g\|_{W_{p}^{l}(-\infty,+\infty)} .
\end{aligned}
$$

Складывая это неравенство с очевидным неравенством

$$
\left\|g^{(l)}\right\|_{L_{p}(-\infty,+\infty)} \leqslant\|g\|_{W_{p}^{l}(-\infty,+\infty)},
$$

получим

$$
\begin{aligned}
\left\|g^{(l-1)}\right\|_{W_{p}^{1}(-\infty,+\infty)} & =\left\|g^{(l-1)}\right\|_{L_{p}(-\infty,+\infty)}+\left\|g^{(l)}\right\|_{L_{p}(-\infty,+\infty)} \\
& \leqslant\left(\frac{\pi}{2}+1\right)\|g\|_{W_{p}^{l}(-\infty,+\infty)} .
\end{aligned}
$$

Из неравенства (26) теперь следует

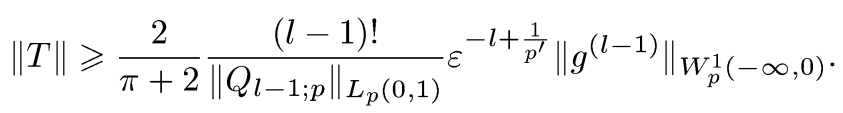

Так как $g^{(l-1)}(-0)=1$, то отсюда вытекает

$$
\|T\| \geqslant \frac{2}{\pi+2} \frac{(l-1) !}{\left\|Q_{l-1 ; p}\right\|_{L_{p}(0,1)}} \varepsilon^{-l+\frac{1}{p^{\prime}}} \inf _{h(-0)=1}\|h\|_{W_{p}^{1}(-\infty, 0)} .
$$

Поскольку для любых рассматриваемых функций $h$

$$
1=|h(-0)| \leqslant \int_{-1}^{0}|h(x)| d x+\int_{-1}^{0}\left|h^{\prime}(x)\right| d x \leqslant\|h\|_{W_{p}^{1}(-\infty, 0)},
$$

Tо

$$
\|T\| \geqslant \frac{2}{\pi+2} \frac{(l-1) !}{\left\|Q_{l-1 ; p}\right\|_{L_{p}(0,1)}} \varepsilon^{-l+\frac{1}{p^{\prime}}} .
$$

Неравенство (25) следует из (27), поскольку (см. [8])

$$
\left\|Q_{l-1 ; p}\right\|_{L_{p}(0,1)} \leqslant\left\|Q_{l-1 ; \infty}\right\|_{L_{\infty}(0,1)}=2 \cdot 4^{-l+1} .
$$

Обозначим теперь для $l, n \in \mathbb{N}$ и $1 \leqslant p \leqslant+\infty$ через $M_{l, n, p}$ множество таких $1 \leqslant q \leqslant+\infty$ и $\beta \in \mathbb{N}_{0}^{n}$, что для некоторого $A>0$ для любых $f \in W_{p}^{l}\left(\mathbb{R}^{n}\right)$ выполняется неравенство

$$
\left\|D^{\beta} f\right\|_{L_{q}\left(\mathbb{R}^{n}\right)} \leqslant A\|f\|_{W_{p}^{l}\left(\mathbb{R}^{n}\right)} .
$$

Как известно, это означает, что или $p \leqslant q<+\infty$ и $|\beta| \leqslant l-n\left(\frac{1}{p}-\frac{1}{q}\right)$ или $q=+\infty$ и для $p=+\infty|\beta| \leqslant l$, для $1<p<+\infty|\beta|<l-\frac{n}{p}$, для $p=1|\beta| \leqslant l-n$. Обозначим далее для открытого множества $\Omega \subset \mathbb{R}^{n}$ и для $(q, \beta) \in M_{l, n, p}$ через $C^{*}(\Omega, p, q, l, \beta)$ наименьшую постоянную в неравенстве

$$
\left\|D^{\beta} f\right\|_{L_{q}(\Omega)} \leqslant C\|f\|_{W_{p}^{l}(\Omega)} .
$$


Лемма 6. Пусть $l, n \in \mathbb{N}, 1 \leqslant p \leqslant+\infty,(q, \beta) \in M_{l, n, p} u \Omega \subset \mathbb{R}^{n}$ - открытое множество. Тогда норма любого оператора продолжения $T: W_{p}^{l}(\Omega) \rightarrow$ $W_{p}^{l}\left(\mathbb{R}^{n}\right)$ удовлетворяет неравенству

$$
\|T\|_{W_{p}^{l}(\Omega) \rightarrow W_{p}^{l}\left(\mathbb{R}^{n}\right)} \geqslant \sup _{(q, \beta) \in M_{l, n, p}} \frac{C^{*}(\Omega, p, q, l, \beta)}{C^{*}\left(\mathbb{R}^{n}, p, q, l, \beta\right)} .
$$

ДокАЗАТЕЛЬСТво. Будем доказывать неравенство (30) для $\Omega \neq \mathbb{R}^{n}$, исходя из неравенства $(29)$ с точной постоянной с помощью произвольного оператора продолжения $T: W_{p}^{l}(\Omega) \rightarrow W_{p}^{l}\left(\mathbb{R}^{n}\right)$. Тогда

$$
\begin{aligned}
\left\|D^{\beta} f\right\|_{L_{q}(\Omega)} & \leqslant\left\|D^{\beta}(T f)\right\|_{L_{q}\left(\mathbb{R}^{n}\right)} \leqslant C^{*}\left(\mathbb{R}^{n}, p, q, l, \beta\right)\|T f\|_{W_{p}^{l}\left(\mathbb{R}^{n}\right)} \\
& \leqslant C^{*}\left(\mathbb{R}^{n}, p, q, l, \beta\right)\|T\|_{W_{p}^{l}(\Omega) \rightarrow W_{p}^{l}\left(\mathbb{R}^{n}\right)}\|f\|_{W_{p}^{l}(\Omega)} .
\end{aligned}
$$

Значит,

$$
C^{*}\left(\Omega^{n}, p, q, l, \beta\right) \leqslant C^{*}\left(\mathbb{R}^{n}, p, q, l, \beta\right)\|T\|_{W_{p}^{l}(\Omega) \rightarrow W_{p}^{l}\left(\mathbb{R}^{n}\right)},
$$

откуда и следует неравенство (31).

ВТороЕ доКАЗАТЕЛЬСТво. Отметим прежде всего, что при любых $1 \leqslant$ $p \leqslant+\infty, l \in \mathbb{N}$ для любых $f \in W_{p}^{l}(-\infty,+\infty)$ справедливо неравенство

$$
\left\|f^{(l-1)}\right\|_{L_{\infty}(-\infty,+\infty)} \leqslant \sqrt{2 \pi}\|f\|_{W_{p}^{l}(-\infty,+\infty)} .
$$

Оно следует из неравенства ${ }^{1}$

$$
\left\|f^{\prime}\right\|_{L_{\infty}(-\infty,+\infty)} \leqslant 2\|f\|_{L_{p}(-\infty,+\infty)}^{\frac{1}{2}\left(1-\frac{1}{p}\right)}\left\|f^{\prime \prime}\right\|_{L_{p}(-\infty,+\infty)}^{\frac{1}{2}\left(1+\frac{1}{p}\right)},
$$

где $1 \leqslant p \leqslant+\infty$, и использованного в первом доказательстве леммы 5 неравенства Колмогорова-Стейна.

Действительно,

$$
\begin{aligned}
&\left\|f^{(l-1)}\right\|_{L_{\infty}(-\infty,+\infty)} \leqslant 2\left\|f^{(l-2)}\right\|_{L_{p}(-\infty,+\infty)}^{\frac{1}{2}\left(1-\frac{1}{p}\right)}\left\|f^{(l)}\right\|_{L_{p}(-\infty,+\infty)}^{\frac{1}{2}\left(1+\frac{1}{p}\right)} \\
& \leqslant 2\left(\frac{\pi}{2}\|f\|_{L_{p}(-\infty,+\infty)}^{\frac{2}{l}}\left\|f^{(l)}\right\|_{L_{p}(-\infty,+\infty)}^{1-\frac{2}{l}}\right)^{\frac{1}{2}\left(1-\frac{1}{p}\right)}\left\|f^{(l)}\right\|_{L_{p}(-\infty,+\infty)}^{\frac{1}{2}\left(1+\frac{1}{p}\right)} \\
& \leqslant \sqrt{2 \pi}\left(\frac{\pi}{2}\right)^{-\frac{1}{p}}\|f\|_{L_{p}(-\infty,+\infty)}^{\frac{1}{l}\left(1-\frac{1}{p}\right)}\left\|f^{(l)}\right\|_{L_{p}(-\infty,+\infty)}^{\frac{1}{l}\left(l-1+\frac{1}{p}\right)} \\
& \leqslant \sqrt{2 \pi}\left(\frac{\pi}{2}\right)^{-\frac{1}{p}}\left(\frac{1}{l}\left(1-\frac{1}{p}\right)\right)^{\frac{1}{l}\left(1-\frac{1}{p}\right)}\left(\frac{1}{l}\left(l-1+\frac{1}{p}\right)\right)^{\frac{1}{l}\left(l-1+\frac{1}{p}\right)} \\
& \times\left(\|f\|_{L_{p}(-\infty,+\infty)}+\left\|f^{(l)}\right\|_{L_{p}(-\infty,+\infty)},\right.
\end{aligned}
$$

\footnotetext{
${ }^{1}$ При $p=+\infty$ это классическое неравенство, восходящее к Э. Ландау и Ж. Адамару. Тот факт, что с той же постоянной оно верно и для любых $1 \leqslant p<+\infty$, вытекает из неравенства

$$
\begin{aligned}
\left|f^{\prime}(x)\right| & \leqslant\left(\frac{p+1}{2}\right)^{\frac{1}{p}} \varepsilon^{-1-\frac{1}{p}}\|f\|_{L_{p}(x-\varepsilon, x+\varepsilon)}+2^{-\frac{1}{p}} \varepsilon^{-1-\frac{1}{p}}\left\|f^{\prime \prime}\right\|_{L_{p}(x-\varepsilon, x+\varepsilon)} \\
& \leqslant\left(\frac{p+1}{2}\right)^{\frac{1}{p}} \varepsilon^{-1-\frac{1}{p}}\|f\|_{L_{p}(-\infty,+\infty)}+2^{-\frac{1}{p}} \varepsilon^{-1-\frac{1}{p}}\left\|f^{\prime \prime}\right\|_{L_{p}(-\infty,+\infty)},
\end{aligned}
$$
}

следующего из оценок, полученных в [8], после минимизации по $\varepsilon>0$. 
откуда и следует (31).

С другой стороны, полагая в неравенстве

$$
\left\|f^{(l-1)}\right\|_{L_{\infty}(0, \varepsilon)} \leqslant C^{*}((0, \varepsilon), p, \infty, l, l-1)\|f\|_{W_{p}^{l}(0, \varepsilon)}
$$

$f=\varepsilon^{l-1} Q_{l-1 ; p}\left(\frac{x}{\varepsilon}\right)$, мы получаем

$$
C^{*}((0, \varepsilon), p, \infty, l, l-1) \geqslant \frac{(l-1) !}{\left\|Q_{l-1 ; p}\right\|_{L_{p}(0,1)}} \varepsilon^{-l+\frac{1}{p^{\prime}}}
$$

(в [11] доказано, что при $0<\varepsilon \leqslant 1$ здесь имеет место знак равенства). Следовательно, согласно (31)

$$
\|T\|_{W_{p}^{l}(0, \varepsilon) \rightarrow W_{p}^{l}(-\infty, \infty)} \geqslant \frac{1}{\sqrt{2 \pi}} \frac{(l-1) !}{\left\|Q_{l-1 ; p}\right\|_{L_{p}(0,1)}} \varepsilon^{-l+\frac{1}{p^{\prime}}}
$$

откуда согласно (28) вновь следует (25). В заключение приведем основной результат $\S 3$.

Tеорема 3. Существуют такие числа $C_{12}>0, C_{13}>0$, что для любых $l \in \mathbb{N}, \quad 1 \leqslant p \leqslant+\infty,-\infty \leqslant a<b \leqslant+\infty$ справедливо неравенство

$$
C_{12}^{l}\left(1+\frac{l^{l}}{(b-a)^{l-\frac{1}{p^{\prime}}}}\right) \leqslant \inf _{T: W_{p}^{l}(a, b) \rightarrow W_{p}^{l}(-\infty,+\infty)}\|T\| \leqslant C_{13}^{l}\left(1+\frac{l^{l}}{(b-a)^{l-\frac{1}{p^{\prime}}}}\right) .
$$

ДоКАЗАТЕЛЬСтво. Оценка снизу следует из леммы 5 и очевидного неравенства

$$
\|T\|_{W_{p}^{l}(a, b) \rightarrow W_{p}^{l}(-\infty,+\infty)} \geqslant 1 .
$$

Оценка сверху для $b-a<1$ следует из леммы 4 , а для $b-a \geqslant 1$ из леммы 3 .

\section{§4. Разбиение единицы}

В этом параграфе описывается конструкция специального разбиения единицы и приводятся оценки производных от элементов разбиения единицы.

Пусть для $x \in \mathbb{R}^{n} \bar{x}=\left(x_{1}, \ldots, x_{n-1}\right)$. В этом параграфе мы будем рассматривать области $\Omega \subset \mathbb{R}^{n}$ вида

$$
\Omega=\left\{x \in \mathbb{R}^{n}: x_{n}<\varphi(\bar{x})\right\},
$$

где функция $\varphi$ удовлетворяет условию Липшица, т.е. сушествует число $M>0$ такое, что

$$
|\varphi(\bar{x})-\varphi(\bar{y})| \leqslant M|\bar{x}-\bar{y}|, \quad \bar{x}, \bar{y} \in \mathbb{R}^{n-1} .
$$

$$
\begin{aligned}
\text { Положим } G=\mathbb{R}^{n} \backslash \bar{\Omega}=\left\{x \in \mathbb{R}^{n}, \quad x_{n}>\varphi(\bar{x})\right\} \text { и } \\
G_{m}=\left\{x \in G, 2^{-m-1}<x_{n}-\varphi(\bar{x}) \leqslant 2^{-m}\right\}, \quad m \in \mathbb{Z} .
\end{aligned}
$$


Лемма 7. Существует иисло $C_{1}>0$ и для любъх $n, l \in \mathbb{N}$ существует последовательность неотрицательных функиий $\psi_{m} \in C^{\infty}\left(\mathbb{R}^{n}\right), m \in \mathbb{Z}$ (зависящих от $m, n, l, \varphi)$, удовлетворяющие следующим условиям:

1) $\sum_{m=-\infty}^{+\infty} \psi_{m}(x)= \begin{cases}1, & x \in G, \\ 0, & x \notin G ;\end{cases}$
2) $G=\bigcup_{m=-\infty}^{+\infty} \operatorname{supp} \psi_{m}$,

причем кратность покрытия $\kappa\left(\left\{\operatorname{supp} \psi_{m}\right\}_{m \in \mathbb{Z}}\right)=2$;

3) $G_{m} \subset \operatorname{supp} \psi_{m} \subset G_{m}^{*} \subset G_{m-1} \cup G_{m} \cup G_{m+1}$, $G_{m}^{*}=\left\{x \in G, 2^{-m-1}\left(1-2^{-4}\right)<x_{n}-\varphi(\bar{x}) \leqslant 2^{-m}\left(1+2^{-4}\right)\right\} ;$

4) $\forall \alpha \in \mathbb{N}_{0}^{n}:|\alpha| \leqslant l$

$$
\left|\left(D^{\alpha} \psi_{m}\right)(x)\right| \leqslant\left[C_{1}^{n}(1+M) 2^{m} l\right]^{|\alpha|}, \quad x \in \mathbb{R}^{n} .
$$

ДокАЗАТЕЛЬСТво. Пусть $A_{\delta}$ - оператор усреднения с ядром $\omega$, построенным в теореме 1. Для любого $m \in \mathbb{Z}$ положим

$$
\Gamma_{m}=\left\{x \in G: x_{n}-\varphi(\bar{x})=2^{-m}\right\}, \quad \delta_{m}=(1+M)^{-1} 2^{-m-5},
$$

обозначим через $\chi_{m}$ характеристическую функцию множества $G_{m}$ и определим функцию $\psi_{m}$ следуюшим образом:

$$
\psi_{m}(x)= \begin{cases}\left(A_{\delta_{m}} \chi_{m}\right)(x), & \text { если } x \in\left(\Gamma_{m}\right)^{\delta_{m}}, \\ 1, & \text { если } x \in G_{m} \backslash\left(\left(\Gamma_{m}\right)^{\delta_{m}} \cup\left(\Gamma_{m+1}\right)^{\delta_{m+1}}\right), \\ \left(A_{\delta_{m+1}} \chi_{m}\right)(x), & \text { если } x \in\left(\Gamma_{m+1}\right)^{\delta_{m+1}}, \\ 0 & \text { в остальных случаях. }\end{cases}
$$

Таким образом, функция $\psi_{m}$ представляет собой усреднение характеристической функции $\chi_{m}$ множества $G_{m}$ с шагом $\delta_{m}$ "в сторону множества $G_{m-1}$ " и с шагом $\delta_{m+1}$ "в сторону множества $G_{m+1}$ ". При этом характеристические функции $\chi_{m-1}$ и $\chi_{m}$ соседних множеств $G_{m-1}$ и $G_{m}$ "в месте стыка" усредняются с одинаковым шагом $\delta_{m}$, а характеристические функции $\chi_{m}$ и $\chi_{m+1}$ соседних множеств $G_{m}$ и $G_{m+1}$ "в месте стыка" усредняются также с одинаковым, но уже другим шагом $\delta_{m+1}$ (см. рис. 1$)$.

Из определения функции $\psi_{m}$ следует, что $\psi_{m} \in C^{\infty}\left(\mathbb{R}^{n}\right)$. Бесконечно непрерывная дифференцируемость в точках $x \in \partial\left(\left(\Gamma_{m}\right)^{\delta_{m}}\right)$ следует из того, что для достаточно малого $\varepsilon>0$ для любого $y \in B(x, \varepsilon) \psi_{m}(y)=\left(A_{\delta_{m}} \chi_{m}\right)(y)$, при этом $\left(D^{\alpha} \psi_{m}\right)(x)=0$; так же рассматривается случай, когда $x \in \partial\left(\left(\Gamma_{m+1}\right)^{\delta_{m+1}}\right)$. Кроме того,

$$
G_{m} \subset \operatorname{supp} \psi_{m} \subset\left(G_{m}\right)^{\delta_{m}} \subset G_{m}^{*} .
$$

Поясним последнее включение. Если $u \in\left(G_{m}\right)^{\delta_{m}}$, то существует такое $x \in G_{m}$, что $u \in B\left(x, \delta_{m}\right)$, поэтому

$$
\begin{aligned}
u_{n}-\varphi(\bar{u}) & =u_{n}-x_{n}+x_{n}-\varphi(\bar{x})+\varphi(\bar{x})-\varphi(\bar{u}) \\
& >-\delta_{m}+2^{-m-1}-M \delta_{m}>2^{-m-1}\left(1-2^{-4}\right) .
\end{aligned}
$$




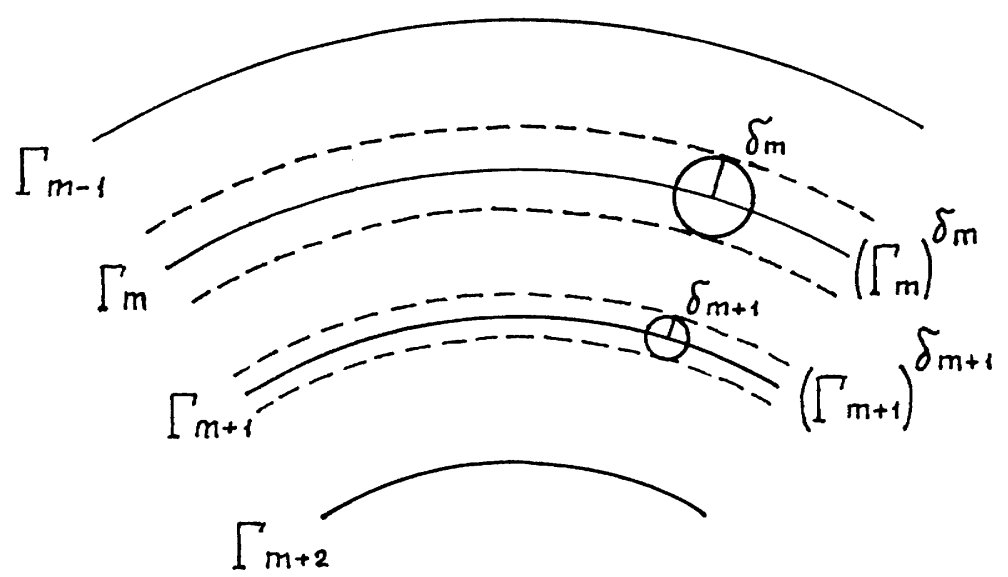

Рис. 1

Аналогично доказывается, что $u_{n}-\varphi(\bar{u}) \leqslant 2^{-m}\left(1-2^{-4}\right)$.

Докажем 1). Если $x \notin G$, то все $\psi_{m}(x)=0$ и $\sum_{m=-\infty}^{+\infty} \psi_{m}(x)=0$. Пусть $x \in G$; тогда сушествует такое $k=k(x)$, что $x \in G_{k}$. Если $x \in G_{k} \backslash\left(\left(\Gamma_{k}\right)^{\delta_{k}} \cup\left(\Gamma_{k+1}\right)^{\delta_{k+1}}\right)$, то $\psi_{k}(x)=1$, а при $m \neq k \quad \psi_{m}(x)=0$ и $\sum_{m=-\infty}^{+\infty} \psi_{m}(x)=1$. Если $x \in G_{k} \cap$ $\left(\Gamma_{k+1}\right)^{\delta_{k+1}}$, то для $m \neq k, k+1 \psi_{m}(x)=0$ и

$$
\begin{aligned}
\sum_{m=-\infty}^{+\infty} \psi_{m}(x) & =\psi_{k}(x)+\psi_{k+1}(x)=\left(A_{\delta_{k+1}} \chi_{k}\right)(x)+\left(A_{\delta_{k+1}} \chi_{k+1}\right)(x) \\
& =\left(A_{\delta_{k+1}}\left(\chi_{k}+\chi_{k+1}\right)\right)(x)=\left(A_{\delta_{k+1}} \chi_{G_{k} \cup G_{k+1}}\right)(x)=1,
\end{aligned}
$$

поскольку $x \in B\left(x, \delta_{k+1}\right) \subset G_{k} \cup G_{k+1}$. Аналогично рассматривается случай, когда $x \in G_{k} \cap\left(\Gamma_{k}\right)^{\delta_{k}}$.

Получим неравенство 4). Достаточно рассмотреть точки $x \in\left(\Gamma_{m}\right)^{\delta_{m}} \cup$ $\left(\Gamma_{m+1}\right)^{\delta_{m+1}}$. Если $x \in\left(\Gamma_{m}\right)^{\delta_{m}}$, то

$$
\begin{aligned}
\left|\left(D^{\alpha} \psi_{m}\right)(x)\right| & =\left|D^{\alpha}\left(\omega_{\delta_{m}} * \chi_{m}\right)(x)\right|=\delta_{m}^{-|\alpha|}\left|\left(\left(D^{\alpha} \omega\right)_{\delta_{m}} * \chi_{m}\right)(x)\right| \\
& \leqslant \delta_{m}^{-|\alpha|}\left\|\left(D^{\alpha} \omega\right)_{\delta_{m}}\right\|_{L_{1}\left(\mathbb{R}^{n}\right)}=\delta_{m}^{-|\alpha|}\left\|D^{\alpha} \omega\right\|_{L_{1}\left(B_{1}\right)} \\
& \leqslant \delta_{m}^{-|\alpha|} v_{n}\left\|D^{\alpha} \omega\right\|_{L_{\infty}\left(\mathbb{R}^{n}\right)} \leqslant\left(\left(2 \widetilde{C}_{1}\right)^{n} 2^{5}(1+M) 2^{m} l\right)^{|\alpha|}
\end{aligned}
$$

где $\widetilde{C}_{1}$ - постоянная из неравенства (3) из $\S 1$ (обозначенная там через $C_{1}$ ). Аналогичная оценка справедлива и для $x \in\left(\Gamma_{m+1}\right)^{\delta_{m+1}}$ с заменой $2^{5}$ на $2^{6}$, откуда и следует неравенство (4) с $C_{1}=2^{7} \widetilde{C}_{1}$. 
§5. Двусторонняя оценка точной постоянной в неравенстве для $L_{p}$-нормы промежуточной производной

Для получения оценки сверху нам понадобятся несколько лемм, связанных с понятием множества, удовлетворяющего условию конуса.

ОПРЕДЕЛЕНИЕ 1. Открытое множество $\Omega \subset \mathbb{R}^{n}$ удовлетворяет условию конуса (с параметрами $r>0$ и $h>0$ ), если сушествует такой конус

$$
K=\left\{y \in \mathbb{R}^{n}: 0<\left(\sum_{i=1}^{n-1} y_{i}^{2}\right)^{\frac{1}{2}}<\frac{r}{h} y_{n}<r\right\}
$$

что для любого $x \in \Omega$ существует конус $K_{x} \subset \Omega$ с вершиной в точке $x$, конгруэнтньй конусу $K$.

Пусть $B$ - открытый шар, $(x, y)$ - интервал с конщами в точках $x, y \in \mathbb{R}^{n}$. Назовем коническим телом с верииной в точке $x \in \mathbb{R}^{n}$, построенным по шару $B$, множество

$$
V_{x}=\bigcup_{y \in B}(x, y)
$$

(если $x \in B$, то $V_{x}=B$ ).

ОПРЕДЕЛЕНИЕ 2 . Область $\Omega \subset \mathbb{R}^{n}$ звездна относительно шара $B \subset \Omega$, если для любого $x \in \Omega V_{x} \subset \Omega$.

Впишем в конус $K_{x}$ открытый шар $B\left(y(x), r_{1}\right)\left(\right.$ его радиус $\left.r_{1}=\frac{r h}{r+\sqrt{r^{2}+h^{2}}}\right)$ и рассмотрим коническое тело с вершиной в точке $x$, построенное по шару $B\left(y(x), r_{1}\right)$. Обозначим его через $\widetilde{K}_{x}$ и положим $h_{1}=|x-y(x)|=h-r_{1}$.

Ниже мы будем пользоваться следуюшим неравенством: если $E_{k} \subset \mathbb{R}^{n}$, где $k \in\{1, \ldots, m\}, m \in \mathbb{N}$ или $m=+\infty,-$ измеримые множества, $E=\bigcup_{k=1}^{m} E_{k}$ и кратность покрытия $\left\{E_{k}\right\}_{k=1}^{m}$ равна $\kappa$, то для любой неотрищательной измеримой на $E$ функции $\varphi$

$$
\sum_{k=1}^{m} \int_{E_{k}} \varphi(x) d x \leqslant \kappa \int_{E} \varphi(x) d x
$$

в частности,

$$
\sum_{k=1}^{m} \operatorname{mes} E_{k} \leqslant \kappa \operatorname{mes} E \text {. }
$$

Лемма 8. Для любого открытого множества $\Omega \subset \mathbb{R}^{n}$, удовлетворяющего условию конуса с параметрами $r$ u $h$, существуют такие ограниченные области $\Omega_{k} \subset \Omega$, звездные относительно шаров $B_{k} \subset \bar{B}_{k} \subset \Omega_{k}$, әде $k \in\{1, \ldots, m\}$ (для ограниченных $\Omega m \in \mathbb{N}$, а для неограниченных $\Omega m=\infty)$, чmo

1) $\Omega=\bigcup_{k=1}^{m} \Omega_{k}$

2) для любого $k \in\{1, \ldots, m\}$

$$
r_{1}=\operatorname{diam} B_{k} \leqslant \operatorname{diam} \Omega_{k} \leqslant 2 h,
$$


откуда, в частности, следует, что

$\left.2^{\prime}\right)$ для любого $k \in\{1, \ldots, m\}$

$$
\frac{\operatorname{diam} \Omega_{k}}{\operatorname{diam} B_{k}} \leqslant 4\left(1+\frac{h}{r}\right)
$$

3) кратность покрытия

$$
\kappa\left(\left\{\Omega_{k}\right\}_{k=1}^{m}\right) \leqslant 8^{n}\left(1+\frac{h}{r}\right)^{n}
$$

ДокАЗАТЕЛЬСТво. Пусть $x \in \Omega$. Рассмотрим кроме конуса $K_{x}$ и конического тела $\widetilde{K}_{x}$ еще и коническое тело $K_{x}^{*}$ с вершиной в точке $z(x)=x+\frac{\varepsilon_{x}(x-y(x))}{|x-y(x)|}$, где $\varepsilon_{x}=\frac{1}{2} \min \left\{r_{1}, \operatorname{dist}(x, \partial \Omega)\right\}$, построенное по шару $B\left(y(x), r_{1}\right)$. Таким образом, $x \in K_{x}^{*} \subset \Omega$ и $\Omega \subset \bigcup_{x \in \Omega} K_{x}^{*}$ (см. pис. 2).

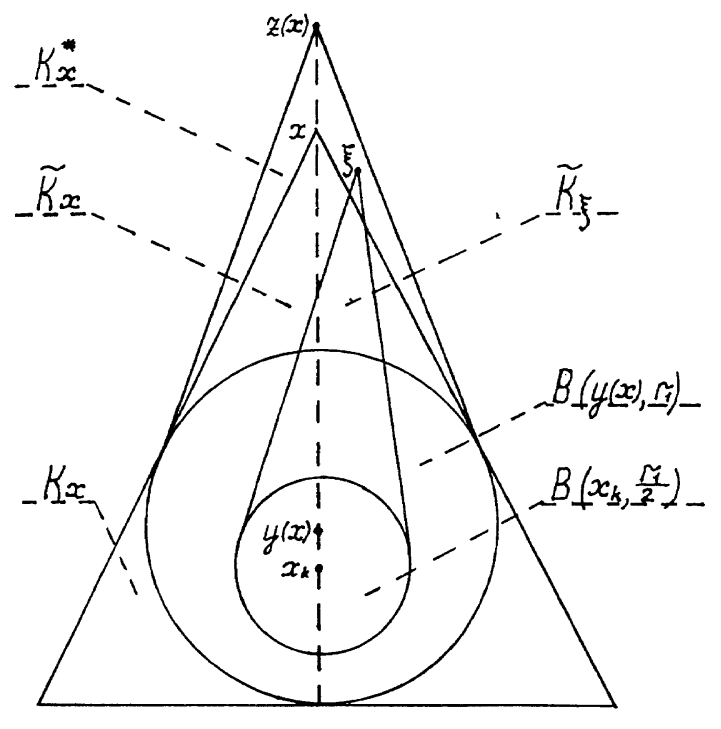

Рис. 2

Обозначим через $\kappa_{n}$ минимальную кратность покрытия $\mathbb{R}^{n}$ шарами единичного радиуса (или, что то же самое, одинакового радиуса). Выберем такие $x_{k} \in \mathbb{R}^{n}$ $(k \in \mathbb{N})$, что $\mathbb{R}^{n}=\bigcup_{k \in \mathbb{N}} B\left(x_{k}, \frac{r_{1}}{2}\right)$ и $\kappa\left(\left\{B\left(x_{k}, \frac{r_{1}}{2}\right)\right\}_{k \in \mathbb{N}}\right)=\kappa_{n}$, и положим

$$
G_{k}=\bigcup_{y(x) \in \Omega \cap B\left(x_{k}, \frac{r_{1}}{2}\right)} K_{x}^{*}
$$

Пусть $G_{k} \neq \varnothing$ и $\xi \in G_{k}$; тогда существует такое $x \in B\left(x_{k}, \frac{r_{1}}{2}\right) \cap \Omega$, что $\xi \in K_{x}^{*}$. Рассмотрим коническое тело $\widetilde{K}_{\xi}$ с вершиной в точке $\xi$, построенное по шару $B\left(x_{k}, \frac{r_{1}}{2}\right)$. Так как $y(x) \in B\left(x_{k}, \frac{r_{1}}{2}\right)$, то $B\left(y(x), r_{1}\right) \supset B\left(x_{k}, \frac{r_{1}}{2}\right)$ и 
$\widetilde{K}_{\xi} \subset K_{x}^{*} \subset \Omega$. Следовательно, множество $G_{k}$ звездно относительно шара $B\left(x_{k}, \frac{r_{1}}{2}\right)$.

Далее, так как

$$
\left|\xi-x_{k}\right| \leqslant\left|z(x)-x_{k}\right|=|z(x)-x|+|x-y(x)|+\left|y(x)-x_{k}\right| \leqslant r_{1}+h_{1}=h,
$$

то $G_{k} \subset B\left(x_{k}, h\right)$ и $\operatorname{diam} G_{k} \leqslant 2 h$.

Рассмотрим множества $G_{k}$ для тех $k$, для которых $G_{k} \neq \varnothing$. Если $\Omega$ ограничено, то их конечное число - обозначим его через $m$; если $\Omega$ неограничено, то их счетное число $(m=+\infty)$. Перенумеруем их и обозначим через $\Omega_{1}, \Omega_{2}, \ldots$ Так как $\Omega \subset$ $\bigcup_{k=1}^{\infty} B\left(x_{k}, \frac{r_{1}}{2}\right)$, то

$$
\Omega=\bigcup_{k=1}^{\infty} G_{k}=\bigcup_{k=1}^{m} \Omega_{k}
$$

Таким образом, для множеств $\Omega_{k}$ выполнены свойства 1$\left.), 2\right), 2^{\prime}$ ). Наконец,

$$
\kappa\left(\left\{\Omega_{k}\right\}_{k=1}^{m}\right) \leqslant \kappa\left(\left\{B\left(x_{k}, h\right)\right\}_{k=1}^{\infty}\right) .
$$

Пусть $x \in \mathbb{R}^{n}$; тогда количество $\kappa(x)$ шаров $B\left(x_{k}, h\right) \ni x$ равно количеству точек $x_{k} \in B(x, h)$. Так как $\kappa\left(\left\{B\left(x_{k}, \frac{r_{1}}{2}\right)\right\}_{k=1}^{\infty}\right)=\kappa_{n}$, то согласно неравенству (2)

$$
\begin{aligned}
\kappa(x) \operatorname{mes} B\left(x_{k}, \frac{r_{1}}{2}\right) & =\sum_{k: B\left(x_{k}, \frac{r_{1}}{2}\right) \ni x} \operatorname{mes} B\left(x_{k}, \frac{r_{1}}{2}\right) \\
& \leqslant \kappa_{n} \operatorname{mes} \bigcup_{k: B\left(x_{k}, \frac{r_{1}}{2}\right) \ni x} B\left(x_{k}, \frac{r_{1}}{2}\right) \leqslant \kappa_{n} \operatorname{mes} B(x, h)
\end{aligned}
$$

и

$$
\kappa(x) \leqslant \kappa_{n} 2^{n}\left(\frac{h}{r_{1}}\right)^{n} \leqslant \kappa_{n} 4^{n}\left(1+\frac{h}{r}\right)^{n},
$$

откуда и следует свойство (3), поскольку $\kappa_{n} \leqslant 2^{n}$.

Отметим, что для случая ограниченного множества $\Omega$ первое утверждение леммы 2 доказано в [14] (по сравнению с [14] рассуждения изменены, что позволяет получить оценки 2) и 3$)$ ).

Лемма 9. Пусть $1 \leqslant p \leqslant \infty, \Omega=\bigcup_{k=1}^{s} \Omega_{k}$, әде $s \in \mathbb{N}$ или $s=\infty, \Omega_{k} \subset \mathbb{R}^{n}-$ измеримые множества, причем при $1 \leqslant p \leqslant+\infty \quad \kappa=\kappa\left(\left\{\Omega_{k}\right\}_{k=1}^{s}\right)<+\infty$. Пусть далее $M \in \mathbb{N}$ или $M=+\infty, C_{m}>0 \quad(m \in\{1, \ldots, M\}), f_{m}$ $(m \in\{1, \ldots, M\})$ и $g$-измеримье на $\Omega$ функиии. Если для любого $k \in\{1, \ldots, s\}$ справедливо неравенство

$$
\|g\|_{L_{p}\left(\Omega_{k}\right)} \leqslant \sum_{m=1}^{M} C_{m}\left\|f_{m}\right\|_{L_{p}\left(\Omega_{k}\right)},
$$

mo

$$
\|g\|_{L_{p}(\Omega)} \leqslant \kappa^{\frac{1}{p}} \sum_{m=1}^{M} C_{m}\|f\|_{L_{p}(\Omega)} .
$$


ДоКАЗАТЕЛЬСТВО. Если $p=+\infty$, то

$$
\begin{aligned}
\|g\|_{L_{\infty}(\Omega)} & =\sup _{k \in\{1, \ldots, s\}}\|g\|_{L_{\infty}\left(\Omega_{k}\right)} \leqslant \sum_{m=1}^{M} C_{m} \sup _{k \in\{1, \ldots, s\}}\left\|f_{m}\right\|_{L_{\infty}\left(\Omega_{k}\right)} \\
& =\sum_{m=1}^{M} C_{m}\left\|f_{m}\right\|_{L_{\infty}(\Omega)} .
\end{aligned}
$$

Пусть теперь $1 \leqslant p<+\infty$. Тогда согласно неравенству Минковского для сумм и неравенству (1)

$$
\begin{aligned}
\|g\|_{L_{p}(\Omega)} & \leqslant\left(\sum_{k=1}^{s}\|g\|_{L_{p}\left(\Omega_{k}\right)}^{p}\right)^{\frac{1}{p}} \leqslant\left(\sum_{k=1}^{s}\left(\sum_{m=1}^{M} C_{m}\left\|f_{m}\right\|_{L_{p}\left(\Omega_{k}\right)}\right)^{p}\right)^{\frac{1}{p}} \\
& \leqslant \sum_{m=1}^{M} C_{m}\left(\sum_{k=1}^{s}\left\|f_{m}\right\|_{L_{p}\left(\Omega_{k}\right)}^{p}\right)^{\frac{1}{p}}=\sum_{m=1}^{M} C_{m}\left(\sum_{k=1}^{s} \int_{\Omega_{k}}\left|f_{m}(x)\right|^{p} d x\right)^{\frac{1}{p}} \\
& \leqslant \kappa^{\frac{1}{p}} \sum_{m=1}^{M} C_{m}\left(\int_{\Omega}\left|f_{m}(x)\right|^{p} d x\right)^{\frac{1}{p}}=\kappa^{\frac{1}{p}} \sum_{m=1}^{M} C_{m}\left\|f_{m}\right\|_{L_{p}(\Omega)}
\end{aligned}
$$

Лемма 10. Существует число $C_{1}>0$ такое, ито для любого $n \in \mathbb{N} u$ для любого ограниченного открытого множества $\Omega \subset \mathbb{R}^{n}($ с диаметром $\mathscr{D})$, звездного относительно шара $B$ ( с диаметром $d)$, для любьх $l \in \mathbb{N}, 1 \leqslant p \leqslant$ $+\infty, \beta \in \mathbb{N}_{0}^{n}:|\beta|<l, f \in W_{p}^{l}(\Omega)$ верно следующее неравенство:

$$
\begin{aligned}
\frac{\left\|D^{\beta} f\right\|_{L_{p}(\Omega)}}{(\operatorname{mes} \Omega)^{\frac{1}{p}}} \leqslant C_{1}^{n l} & {\left[\left(\frac{|\beta|}{\mathscr{D}}\right)^{|\beta|}\left(\frac{\mathscr{D}}{d}\right)^{l-1} \frac{\|f\|_{L_{p}(B)}}{(\operatorname{mes} B)^{\frac{1}{p}}}\right.} \\
+ & \left.\left(\frac{\mathscr{D}}{l-|\beta|}\right)^{l-|\beta|}\left(\frac{\mathscr{D}}{d}\right)^{n-1} \frac{\|f\|_{w_{p}^{l}(\Omega)}}{(\operatorname{mes} \Omega)^{\frac{1}{p}}}\right] .
\end{aligned}
$$

ДоКАЗАТЕЛЬСтво. Пусть $B=B\left(x_{0}, \frac{d}{2}\right) \subset \Omega$ - шар звездности. Для любой функции $f \in W_{p}^{l}(\Omega)$ справедливо интегральное представление [14], [24], [23]: для почти всех $x \in \Omega$

$$
\begin{aligned}
\left(D^{\beta} f\right)(x)= & \int_{B}\left[\sum_{|\alpha|<l-|\beta|} \frac{(-1)^{|\alpha|+|\beta|}}{\alpha !} D_{y}^{\alpha+\beta}\left((x-y)^{\alpha} \omega(y)\right) f(y)\right] d y \\
& +(l-|\beta|) \sum_{|\alpha|=l-|\beta|} \frac{1}{\alpha !} \int_{V_{x}} \frac{\left(D^{\alpha+\beta} f\right)(y)}{|x-y|^{n-l+|\beta|}} \frac{(x-y)^{\alpha}}{|x-y|^{l-|\beta|}} w(x, y) d y
\end{aligned}
$$

где $V_{x}$ - коническое тело, определенное выше,

$$
\begin{gathered}
w(x, y)=\int_{|x-y|}^{+\infty} \omega\left(x+\varrho \frac{x-y}{|x-y|}\right) \varrho^{n-1} d \varrho, \\
\omega \in C_{0}^{\infty}\left(\mathbb{R}^{n}\right), \quad \operatorname{supp} \omega \subset \bar{B}, \quad \int_{B} \omega(x) d x=1 .
\end{gathered}
$$


Выберем в качестве $\omega$ функцию

$$
\omega(x)=\left(\frac{2}{d}\right)^{n} \widetilde{\omega}\left(\frac{2}{d}\left(x-x_{0}\right)\right)
$$

где $\widetilde{\omega}-$ функция из теоремы 1 .

Учитывая неравенство 4) из теоремы 1 , получим, что

$$
\begin{aligned}
& \left|D_{y}^{\alpha+\beta}\left((x-y)^{\alpha} \omega(y)\right)\right| \leqslant \sum_{0 \leqslant \gamma \leqslant \alpha+\beta} C_{\alpha+\beta}^{\gamma}\left|D_{y}^{\gamma}(x-y)^{\alpha}\right|\left|\left(D^{\alpha+\beta-\gamma} \omega\right)(y)\right| \\
& \quad \leqslant \sum_{0 \leqslant \gamma \leqslant \alpha} C_{\alpha+\beta}^{\gamma} \frac{\alpha !}{(\alpha-\gamma) !}\left|(x-y)^{\alpha-\gamma}\right|\left(\frac{2}{d}\right)^{n+|\alpha+\beta-\gamma|} 2^{n} v_{n}^{-1} C_{2}^{|\alpha+\beta-\gamma| n} l^{|\alpha+\beta-\gamma|} \\
& \quad \leqslant 2^{2 n+l} \alpha ! C_{2}^{l n} v_{n}^{-1} l^{|\beta|} d^{-n} n^{l} \mathscr{D}^{-|\beta|}\left(\frac{\mathscr{D}}{d}\right)^{|\alpha|+|\beta|} \sum_{0 \leqslant \gamma \leqslant \alpha} \frac{l^{|\alpha-\gamma|}}{(\alpha-\gamma) !} \\
& \leqslant\left(16 e C_{2}\right)^{n l} v_{n}^{-1} \alpha ! l^{|\beta|} d^{-n} \mathscr{D}^{-|\beta|}\left(\frac{\mathscr{D}}{d}\right)^{|\alpha|+|\beta|} .
\end{aligned}
$$

Здесь $C_{2} \geqslant 1-$ постоянная из неравенства 4$)$ теоремы $1, \alpha !=\alpha_{1} ! \ldots \alpha_{n} !, C_{\alpha}^{\gamma}=$ $\frac{\alpha !}{\gamma !(\alpha-\gamma) !} ; 0 \leqslant \gamma \leqslant \alpha$ означает, что $0 \leqslant \gamma_{j} \leqslant \alpha_{j}, j=1, \ldots, n$. Мы учли, что

$$
\left|(x-y)^{\alpha-\gamma}\right| d^{-n-|\alpha+\beta-\gamma|} \leqslant d^{-n} \mathscr{D}^{-|\alpha+\beta-\gamma|} \leqslant d^{-n} \mathscr{D}^{-|\beta|}\left(\frac{\mathscr{D}}{d}\right)^{|\alpha+\beta|} .
$$

Так как $\left|\frac{(x-y)^{\alpha}}{|x-y|^{l-|\beta|}}\right| \leqslant 1$ при $|\alpha|=l-|\beta|$, то из свойств 1$\left.), 4\right)$ функции $\widetilde{\omega}$ из теоремы 1 имеем неравенство

$$
\begin{aligned}
\left|\frac{(x-y)^{\alpha}}{|x-y|^{l-|\beta|}} w(x, y)\right| & \leqslant\left(\frac{4}{d}\right)^{n} v_{n}^{-1} \int_{d_{1}}^{d_{2}} \varrho^{n-1} d \varrho \\
& \leqslant\left(\frac{4}{d}\right)^{n} v_{n}^{-1} d \mathscr{D}^{n-1} \leqslant 4^{n} v_{n}^{-1}\left(\frac{\mathscr{D}}{d}\right)^{n-1}
\end{aligned}
$$

Здесь $d_{1}$ - расстояние от точки $x$ до ближайшей точки пересечения прямой, проходяшей через точки $x$ и $y$, со сферой $\partial B$, a $d_{2}-$ расстояние от точки $x$ до другой точки пересечения.

Из неравенства (4), (6), (7), применяя неравенство Минковского, приходим к неравенству

$$
\begin{aligned}
&\left\|D^{\beta} f\right\|_{L_{p}(\Omega)} \leqslant C_{3}^{l n} v_{n}^{-1}\left(\frac{l}{\mathscr{D}}\right)^{|\beta|} d^{-n}\left(\frac{\mathscr{D}}{d}\right)^{l-1}(\operatorname{mes} \Omega)^{\frac{1}{p}}\|f\|_{L_{1}(B)} \\
&+(l-|\beta|) 4^{n} v_{n}^{-1}\left(\frac{\mathscr{D}}{d}\right)^{n-1} \sum_{|\alpha|=l-|\beta|} \frac{1}{\alpha !}\left\|\int_{V_{x}} \frac{\left(D^{\alpha+\beta} f\right)(y)}{|x-y|^{n-l+|\beta|}} d y\right\|_{L_{p}(\Omega)}
\end{aligned}
$$

где $C_{3}=32 e C_{2}$. 
Из неравенства Гёльдера для первого слагаемого и неравенства Юнга для второго слагаемого получаем неравенство

$$
\begin{aligned}
& \left\|D^{\beta} f\right\|_{L_{p}(\Omega)} \leqslant C_{3}^{l n} v_{n}^{-1}\left(\frac{l}{\mathscr{D}}\right)^{|\beta|} d^{-n}\left(\frac{\mathscr{D}}{d}\right)^{l-1}(\operatorname{mes} \Omega)^{\frac{1}{p}}(\operatorname{mes} B)^{\frac{1}{p^{\prime}}}\|f\|_{L_{p}(B)} \\
& \quad+(l-|\beta|) 4^{n} v_{n}^{-1}\left(\frac{\mathscr{D}}{d}\right)^{n-1} \sum_{|\alpha|=l-|\beta|} \frac{1}{\alpha !}\left\|D^{\alpha+\beta} f\right\|_{L_{p}(\Omega)}\left\|\frac{1}{|x|^{n-l+|\beta|} \|}\right\|_{L_{1}(\Omega-\Omega)},
\end{aligned}
$$

где $A-B$ - арифметическая разность множеств $A$ и $B$. Так как

$$
\begin{gathered}
\left\||x|^{-n+l-|\beta|}\right\|_{L_{1}(\Omega-\Omega)} \leqslant \frac{n v_{n} \mathscr{D}^{l-|\beta|}}{l-|\beta|}, \\
l^{|\beta|} \leqslant e^{n l} \beta ! \leqslant e^{n l}|\beta|^{|\beta|}, \quad \frac{1}{\alpha !} \leqslant \frac{e^{l n}}{l^{|\alpha|}} \leqslant \frac{e^{l n}}{(l-|\beta|)^{l-|\beta|}},
\end{gathered}
$$

то

$$
\begin{aligned}
\left\|D^{\beta} f\right\|_{L_{p}(\Omega)} \leqslant & \left(C_{3} e\right)^{l n}(\operatorname{mes} \Omega)^{\frac{1}{p}}\left(\frac{|\beta|}{\mathscr{D}}\right)^{|\beta|}\left(\frac{\mathscr{D}}{d}\right)^{l-1} \frac{\|f\|_{L_{p}(B)}}{(\operatorname{mes} B)^{\frac{1}{p}}} \\
& +n(4 e)^{l n}\left(\frac{\mathscr{D}}{l-|\beta|}\right)^{l-|\beta|}\left(\frac{\mathscr{D}}{d}\right)^{n-1}\|f\|_{w_{p}^{l}(\Omega)} .
\end{aligned}
$$

Лемма доказана с константой $C_{1}=32 e^{2} C_{2}$.

СлЕДСТВИЕ. Если в условиях леммы 6 множество $\Omega$ есть шар $B=B\left(0, \frac{\mathscr{D}}{2}\right)$, то справедливо неравенство

$$
\left\|D^{\beta} f\right\|_{L_{p}(B)} \leqslant C_{1}^{l n}\left[\left(\frac{|\beta|}{\mathscr{D}}\right)^{|\beta|}\|f\|_{L_{p}(B)}+\left(\frac{\mathscr{D}}{l-|\beta|}\right)^{l-|\beta|}\|f\|_{w_{p}^{l}(B)}\right]
$$

(это неравенство является обобщением неравенства (15) из §3 на многомерный случай).

Рассмотрим теперь неравенство (30) для промежуточной производной для случая $p=q$

$$
\left\|D^{\beta} f\right\|_{L_{p}(\Omega)} \leqslant C^{*}(\Omega, p, p, l, \beta)\|f\|_{W_{p}^{l}(\Omega)}
$$

с точной постоянной $C^{*}(\Omega, p, p, l, \beta)$.

Оценку постоянной $C^{*}(\Omega, p, p, l, \beta)$ сверху (а в случае, когда $\Omega$ - ограниченное множество, и снизу) дает

Tеорема 4. Для любых $n \in \mathbb{N}, r, h>0$ cуществует число $C_{4}(n, r, h)>0$ такое, что для любого открытого множества $\Omega \subset \mathbb{R}^{n}$, удовлетворяющего условию конуса с параметрами $r, h$, для любых $l \in \mathbb{N}, \quad \beta \in \mathbb{N}_{0}^{n}: \quad|\beta|<l$, $1 \leqslant p \leqslant+\infty$

$$
C^{*}(\Omega, p, p, l, \beta) \leqslant C_{4}(n, r, h)^{l} l^{|\beta|} .
$$

Если, кроме того, множество $\Omega$ ограничено и имеет диаметр $\mathscr{D}$, то существует такое число $C_{5}(n, \mathscr{D})>0$, что справедливо двустороннее неравенство

$$
C_{5}(n, \mathscr{D})^{l} l^{|\beta|} \leqslant C^{*}(\Omega, p, p, l, \beta) \leqslant C_{4}(n, r, h)^{l} l^{|\beta|} .
$$


ДоКАЗАТЕЛЬСТво. По лемме 8 множество $\Omega$ представляется в виде $\Omega=$ $\bigcup_{k=1}^{m} \Omega_{k}$, где $\Omega_{k}$ - область, звездная относительно шара $B_{k}$. Пусть $\mathscr{D}_{k}, d_{k}-$ диаметры множеств $\Omega_{k}$ и $B_{k}$ соответственно. Согласно лемме 10 для любого $k \in\{1, \ldots, m\} \quad(m \in \mathbb{N}$ или $m=+\infty)$ справедливо неравенство

$$
\begin{aligned}
\left\|D^{\beta} f\right\|_{L_{p}\left(\Omega_{k}\right) \leqslant} \leqslant & C_{1}^{n l}\left[\left(\frac{l}{\mathscr{D}_{k}}\right)^{|\beta|}\left(\frac{\mathscr{D}_{k}}{d_{k}}\right)^{l-1}\left(\frac{\operatorname{mes} \Omega_{k}}{\operatorname{mes} B_{k}}\right)^{\frac{1}{p}}\|f\|_{L_{p}\left(\Omega_{k}\right)}\right. \\
& \left.+\left(\frac{\mathscr{D}_{k}}{l-|\beta|}\right)^{l-|\beta|}\left(\frac{\mathscr{D}_{k}}{d_{k}}\right)^{n-1}\|f\|_{w_{p}^{l}\left(\Omega_{k}\right)}\right] .
\end{aligned}
$$

Из леммы 8 следует, что $\frac{\mathscr{D}_{k}}{d_{k}} \leqslant 4\left(1+\frac{h}{r}\right)$ и $(2 h)^{-1} \leqslant \mathscr{D}_{k}^{-1} \leqslant 2\left(r^{-1}+h^{-1}\right)$. Поэтому из (14) следует, что

$$
\begin{aligned}
\left\|D^{\beta} f\right\|_{L_{p}\left(\Omega_{k}\right)} \leqslant & C_{1}^{n l}\left[l^{|\beta|}\left(2\left(r^{-1}+h^{-1}\right)\right)^{|\beta|} 4^{l-1+\frac{n}{p}}\left(1+\frac{h}{r}\right)^{l-1+\frac{n}{p}}\|f\|_{L_{p}\left(\Omega_{k}\right)}\right. \\
& \left.+4^{n-1}\left(1+\frac{h}{r}\right)^{n-1}\left(\frac{2 h}{l-|\beta|}\right)^{l-|\beta|}\|f\|_{w_{p}^{l}\left(\Omega_{k}\right)}\right]
\end{aligned}
$$

Применяя к (15) лемму 9 и учитывая, что согласно лемме $8 \kappa \leqslant 8^{n}\left(1+\frac{h}{r}\right)^{n}$, получаем неравенство

$$
\begin{gathered}
\left\|D^{\beta} f\right\|_{L_{p}(\Omega)} \leqslant\left(256 C_{1}\right)^{n l}\left(1+\frac{h}{r}\right)^{n-1}\left[l^{|\beta|}\left(r^{-1}+h^{-1}\right)^{|\beta|}\left(1+\frac{h}{r}\right)^{l}\|f\|_{L_{p}(\Omega)}\right. \\
\left.+\left(\frac{h}{l-|\beta|}\right)^{l-|\beta|}\|f\|_{w_{p}^{l}(\Omega)}\right]
\end{gathered}
$$

откуда и следует (12).

Оценка снизу (13) для ограниченного множества $\Omega$ получается подстановкой в неравенство (11) функции $f(x)=\left(x-x_{0}\right)^{\beta}$, где $x_{0} \in \Omega$, и применением неравенства $l^{|\beta|} e^{-n l} \leqslant \beta !:$

$$
C^{*}(\Omega, p, p, l, \beta) \geqslant \frac{\beta !(\operatorname{mes} \Omega)^{\frac{1}{p}}}{\left\|\left(x-x_{0}\right)^{\beta}\right\|_{L_{p}(\Omega)}} \geqslant \frac{\beta !}{\mathscr{D}^{|\beta|}} \geqslant l^{|\beta|}\left[e^{-n} \min \left\{\mathscr{D}^{-1}, 1\right\}\right]^{l} .
$$

Теорема доказана.

\section{§6. Оценка сверху минимальной нормы операторов продолжения для специальной липшицевой области}

Пусть $-\infty \leqslant a_{i}<b_{i} \leqslant+\infty, i=1, \ldots, n-1, a_{n}>-\infty$. Специальной липиичевой областью мы будем называть область $\Omega \subset \mathbb{R}^{n}$ вида

$$
\Omega=\left\{a_{i}<x_{i}<b_{i}, \quad i=1, \ldots, n-1 ; \quad a_{n}<x_{n}<\varphi(\bar{x})\right\},
$$

где функция $\varphi$ удовлетворяет условию Липшица с константой $M$, т.е.

$$
|\varphi(\bar{x})-\varphi(\bar{y})| \leqslant M|\bar{x}-\bar{y}|, \quad a_{i}<x_{i}, y_{i}<b_{i}, \quad i=1, \ldots, n-1,
$$


и для некоторого $\varepsilon>0$ выполняется неравенство

$$
\varphi(\bar{x})>a_{n}+\varepsilon, \quad a_{i}<x_{i}<b_{i}, \quad i=1, \ldots, n-1 .
$$

6.1. Оценки усреднений со сдвигами для ядер с моментами, равными нулю. Пусть $0<a<b<+\infty, 0<d<c<+\infty$. Рассмотрим для $m \in \mathbb{Z}$ множества

$$
\widehat{\Omega}_{m}=\left\{a_{i}<x_{i}<b_{i}, \quad i=1, \ldots, n-1 ; \quad-c 2^{-m}<x_{n}-\varphi(\bar{x})<-d 2^{-m}\right\}
$$

$$
\begin{aligned}
\widehat{G}_{m}= & \left\{a_{i}+\frac{b}{a} 2^{-m}<x_{i}<b_{i}-\frac{b}{a} 2^{-m},\right. \\
& \left.i=1, \ldots, n-1 ; a 2^{-m}<x_{n}-\varphi(\bar{x})<b 2^{-m}\right\} .
\end{aligned}
$$

Для достаточно больших $m \widehat{\Omega}_{m} \subset \Omega\left(\right.$ если $\left.c 2^{-m} \leqslant \varepsilon\right)$ и $\widehat{\Omega}_{m} \neq \varnothing($ если $\left.\frac{b}{a} 2^{-m+1} \leqslant \min _{i=1, \ldots, n-1}\left(d_{i}-c_{i}\right)\right)$.

Обозначим для любых $n, l \in \mathbb{N}$ через $\omega$ (зависящее от $n$ и $l$ ) ядро усреднения, удовлетворяющее условиям теоремы 2 . Тогда, в частности, $\omega \in C_{0}^{\infty}(K)$, где $K=$ $\left\{x \in \mathbb{R}^{n}: \frac{1}{2}<x_{i}<1, i=1, \ldots, n\right\}$ и $\forall \alpha \in \mathbb{N}_{0}^{n}:|\alpha| \leqslant l$

$$
\left|D^{\alpha} \omega(x)\right| \leqslant C_{1}^{l n} l^{|\alpha|}, \quad x \in \mathbb{R}^{n},
$$

где $C_{1}-$ абсолютная постоянная.

Рассмотрим для $m \in \mathbb{Z}$ усреднения со "сдвигами"

$$
\Phi_{m}(x)=\int_{\mathbb{R}^{n}} \Phi\left(\bar{x}-2^{-m} \bar{z}, x_{n}-B 2^{-m} z_{n}\right) \omega(z) d z,
$$

"сдвиг” обеспечивается выбором постоянной $B$ и тем, что supp $\omega \subset K$, в частности $\omega(z)=0$, если $z_{n} \leqslant \frac{1}{2}$. Параметры $a, b, c, d, B$ будут подобраны так, что

$$
\text { если } x \in \widehat{G}_{m}, \quad z \in K, \quad \text { то }\left(\bar{x}-2^{-m} \bar{z}, x_{n}-B 2^{-m} z_{n}\right) \in \widehat{\Omega}_{m} .
$$

Это приведет к тому, что при достаточно больших $m$ значения функции $\Phi_{m}$ на множестве $\widehat{G}_{m} \subset \mathbb{R}^{n} \backslash \bar{\Omega}$ будут определяться значениями функций $\Phi$ на множестве $\widehat{\Omega}_{m} \subset \Omega$, “сдвинутом" по отношению к множеству $\widehat{G}_{m}$ вдоль оси $x_{n}$ на величину порядка $2^{-m}$.

ЛЕмма 11. Существует число $C_{2}>0$, для любих $0<a<b<+\infty$ существует число $C_{3}=C_{3}(a, b)>0$, для любих $n \in \mathbb{N}, \quad M \geqslant 0,0<a<b<+\infty$, $d>0$ существуют числа $B=B(n, M, a, b, d)>0 u c=c(n, M, a, b, d)>0$, обладающие следующими свойствами.

При любых $m \in \mathbb{Z}, l \in \mathbb{N}$ для всякой функиии $f \in C^{\infty}(\Omega)$ при $\beta \in \mathbb{N}_{0}^{n}:|\beta| \leqslant l$, $1 \leqslant p \leqslant+\infty$

1) справедливо неравенство

$$
\left\|D^{\beta} \Phi_{m}\right\|_{L_{p}\left(\widehat{G}_{m}\right)} \leqslant C_{2}^{l n}\left\|D^{\beta} \Phi\right\|_{L_{p}\left(\widehat{\Omega}_{m}\right)}
$$

2) существуют такие функиии $F_{\beta}$ (не зависящие от $m$ ), что

$$
\left\|D^{\beta} \Phi_{m}-F_{\beta}\right\|_{L_{p}\left(\widehat{G}_{m}\right)} \leqslant C_{3}^{l n} l^{|\beta|-l}(1+M+d)^{l} 2^{m(|\beta|-l)}\|\Phi\|_{w_{p}^{l, \ldots, l}\left(\widehat{\Omega}_{m}\right)},
$$

$2 \partial e$

$$
\|\Phi\|_{w_{p}^{l, \ldots, l}\left(\widehat{\Omega}_{m}\right)}=\sum_{i=1}^{n}\left\|D_{i}^{l} \Phi\right\|_{L_{p}\left(\widehat{\Omega}_{m}\right)} .
$$


ЗАмечание. Неравенства

$$
\begin{aligned}
\left\|D^{\beta} \Phi_{m}\right\|_{L_{p}\left(\widehat{G}_{m}\right)} & \leqslant \widetilde{C}_{2}\left\|D^{\beta} \Phi\right\|_{L_{p}\left(\widehat{\Omega}_{m}\right)}, \\
\left\|D^{\beta} \Phi_{m}-F_{\beta}\right\|_{L_{p}\left(\widehat{G}_{m}\right)} & \leqslant \widetilde{C}_{3} 2^{m(|\beta|-l)}\|\Phi\|_{w_{p}^{l, \ldots, l}\left(\widehat{\Omega}_{m}\right)},
\end{aligned}
$$

где $\widetilde{C}_{2}$ и $\widetilde{C}_{3}$ не зависят от $\Phi$ и $m$, без оценки зависимости $\widetilde{C}_{2}$ и $\widetilde{C}_{3}$ от $l, M$ и $d$ доказаны в [6] (см. лемму 8).

ДоКАЗАТЕЛЬСТво. Выберем $B$ и $c$ так, чтобы

$$
2 \frac{b}{a}\left(\frac{b}{a} M n+b+d\right) \leqslant B \leqslant 2^{10} \frac{b}{a}\left(\frac{b}{a} M n+b+d\right)
$$

и

$$
c=\frac{b}{a}(M n+2 B) .
$$

Выбор параметров $c$ и $B$ обеспечивает выполнение не только условия (8), но и следующего более сильного условия:

если $x \in \widehat{G}_{m}, \quad \frac{a}{2 b}<z_{j}<\frac{b}{a}, \quad j=1, \ldots, n, \quad$ то $\left(\bar{x}-2^{-m} \bar{z}, x_{n}-B 2^{-m} z_{n}\right) \in \widehat{\Omega}_{m}$.

1. Докажем 1). Пусть сначала $\beta=0$. Согласно (6)

$$
\left|\Phi_{m}(x)\right| \leqslant C_{1}^{l n} \int_{\frac{1}{2}}^{1} \cdots \int_{\frac{1}{2}}^{1}\left|\Phi\left(\bar{x}-2^{-m} \bar{z}, x_{n}-B 2^{-m} z_{n}\right)\right| d z .
$$

Согласно обобщенному неравенству Минковского

$$
\begin{aligned}
\left\|\Phi_{m}\right\|_{L_{p}\left(\widehat{G}_{m}\right)} & \leqslant C_{1}^{l n} \int_{K}\left\|\Phi\left(\bar{x}-2^{-m} \bar{z}, x_{n}-B 2^{-m} z_{n}\right)\right\|_{L_{p}\left(\widehat{G}_{m}\right)} d z \\
& \leqslant C_{1}^{l n} \sup _{z \in K}\left\|\Phi\left(\bar{x}-2^{-m} \bar{z}, x_{n}-B 2^{-m} z_{n}\right)\right\|_{L_{p}\left(\widehat{G}_{m}\right)} \leqslant C_{1}^{l n}\|\Phi\|_{L_{p}\left(\widehat{\Omega}_{m}\right)},
\end{aligned}
$$

поскольку при указанном выборе параметров вьполняется свойство (8). Тем самым доказано неравенство (9).

При $|\beta|>0$ достаточно вьполнить дифференцирование под знаком интеграла $\left(D^{\beta} \Phi_{m}=\left(D^{\beta} \Phi\right)_{m}\right)$ и применить неравенство (9) с $\beta=0$.

2. Докажем неравенство 2). Для функции $\Phi\left(\bar{x}-2^{-m} \bar{z}, x_{n}-B 2^{-m_{n}} z_{n}\right.$ запишем интегральное представление (3) из $\S 2$ (см. лемму 1$)$ :

$$
\begin{aligned}
& \Phi\left(\bar{x}-2^{-m} \bar{z}, x_{n}-B 2^{-m} z_{n}\right)=\int_{\Pi} P\left(\bar{x}-2^{-m} \bar{z}, x_{n}-B 2^{-m} z_{n}, \xi\right) \Phi(\xi) d \xi \\
& \quad+\sum_{i=1}^{n} \int_{\Pi_{i}} Q_{i}\left(\bar{x}-2^{-m} \bar{z}, x_{n}-B 2^{-m} z_{n}, \xi\right) \\
& \quad \times D_{i}^{l} \Phi\left(\xi_{1}, \ldots, \xi_{i}, x_{i+1}-2^{-m} z_{i+1}, \ldots, x_{n}-B 2^{-m} z_{n}\right) d \xi_{1} \ldots d \xi_{i} \\
& \equiv \sum_{i=0}^{n} r_{i}^{(m)}(x, z)
\end{aligned}
$$


где $\Pi_{i}=\left\{y \in \mathbb{R}^{i}: \delta_{j}<y_{j}<\gamma_{j}, j=1, \ldots, i\right\}, \Pi \equiv \Pi_{n}$. Выберем здесь $\delta_{j}$ и $\gamma_{j}$ зависящими от $x$, а именно

$$
\begin{gathered}
\delta_{i}=x_{i}-a^{-1}\left(x_{n}-\varphi(\bar{x})\right), \quad \gamma_{i}=x_{i}-b^{-1}\left(x_{n}-\varphi(\bar{x})\right), \quad i=1, \ldots, n-1, \\
\delta_{n}=x_{n}-B a^{-1}\left(x_{n}-\varphi(\bar{x})\right), \quad \gamma_{n}=x_{n}-\frac{B b^{-1}}{2}\left(x_{n}-\varphi(\bar{x})\right) .
\end{gathered}
$$

Отметим, что так как $x \in \widehat{G}_{m}$, то $\Pi \subset \widehat{\Omega}_{m}$ (см. [6]).

Из (7) и (15) следует, что

$$
D^{\beta} \Phi_{m}(x)=\sum_{i=0}^{n} 2^{m|\beta|} B^{-\beta_{n}} \int_{K}\left(D^{\beta} \omega\right)(z) r_{i}^{(m)}(x, z) d z \equiv \sum_{i=0}^{n} R_{i}^{(m)}(x) .
$$

В качестве $F_{\beta}(x)$, как и в $[6]$, возьмем $R_{0}^{(m)}(x)$. Можно проверить (см. [6]), что $R_{0}^{(m)}$ не зависит от $m$.

3. Из представления $(15)$ для $R_{i}^{(m)}, i=1, \ldots, n$, получаем оценки

$$
\begin{aligned}
\left|R_{i}^{(m)}(x)\right| \leqslant & B^{-\beta_{n}} 2^{m|\beta|} \int_{K}\left[\left|\left(D^{\beta} \omega\right)(z)\right| \int_{\Pi_{i}}\left|Q_{i}\left(\bar{x}-2^{-m} \bar{z}, x_{n}-B 2^{-m} z_{n}, \xi\right)\right|\right. \\
& \left.\times\left|D_{i}^{l} \Phi\left(\xi_{1}, \ldots, \xi_{i}, x_{i+1}-2^{-m} z_{i+1}, \ldots, x_{n}-B 2^{-m} z_{n}\right)\right| d \xi_{1} \ldots d \xi_{i}\right] d z .
\end{aligned}
$$

Так как для $x \in \widehat{G}_{m}$

$$
\begin{gathered}
\gamma_{i}-\delta_{i} \leqslant \frac{b}{a} 2^{-m}, \quad i=1, \ldots, n-1, \quad \gamma_{n}-\delta_{n} \leqslant B \frac{b}{a} 2^{-m}, \\
\left(\gamma_{i}-\delta_{i}\right)^{-1} \leqslant\left(1-\frac{a}{b}\right)^{-1} 2^{m}, \quad i=1, \ldots, n-1
\end{gathered}
$$

то из оценки (2) из $§ 2$ следует

$$
\left|Q_{i}\right| \leqslant C_{4}^{l n}\left(1-\frac{a}{b}\right)^{-i}\left(\frac{b}{a}\right)^{l} l^{-l} B^{l} 2^{m(i-l)}, \quad i=1, \ldots, n
$$

где $C_{4}$ - абсолютная постоянная.

Согласно (6) и (18)

$$
\begin{aligned}
& \left|R_{i}^{(m)}(x)\right| \leqslant C_{5}^{l n} B^{l-\beta_{n}} 2^{m(|\beta|+i-l)} l^{|\beta|-l} \\
& \times \int_{\frac{1}{2}}^{1} \ldots \int_{\frac{1}{2}}^{1}\left(\int_{\Pi_{i}}\left|D_{i}^{l} \Phi\left(\xi_{1}, \ldots, \xi_{i}, x_{i+1}-2^{-m} z_{i+1}, \ldots, x_{n}-B 2^{-m} z_{n}\right)\right| d \xi_{1} \ldots d \xi_{i}\right)_{(19)} d z
\end{aligned}
$$

где $C_{5}=\frac{C_{1} C_{4} b^{2}}{a(b-a)}$.

Интегрируем в (19) по $z_{1}, \ldots, z_{i}$ и производим замену $\xi_{s}=x_{s}-2^{-m_{2}} z_{s}$, $s=1, \ldots, i$. Согласно выбору параметров $\delta_{j}$ и $\gamma_{j}$ по формуле (16) для $x \in \widehat{G}_{m}$ 
переменная $z_{s}=2^{m}\left(x_{s}-\xi_{s}\right)$ изменяется в пределах от $\frac{a}{b}$ до $\frac{b}{a}$ для $s=1, \ldots, n-1$ и от $\frac{a}{2 b}$ до $\frac{b}{a}$ для $s=n$. Следовательно, для $x \in \widehat{G}_{m}$

$$
\left|R_{i}^{(m)}\right| \leqslant C_{5}^{l n} l^{|\beta|-l} 2^{m(|\beta|-l)} B^{l-\beta_{n}} \int_{\frac{a}{2 b}}^{\frac{b}{a}} \ldots \int_{\frac{a}{2 b}}^{\frac{b}{a}}\left|\left(D_{i}^{l} \Phi\right)\left(\bar{x}-2^{-m} \bar{z}, x_{n}-B 2^{-m} z_{n}\right)\right| d z .
$$

Применяя обобщенное неравенство Минковского, как в п. 1, и учитывая, что наряду с (8) выполняется также (13), имеем

$$
\begin{aligned}
\left\|R_{i}^{(m)}\right\|_{L_{p}\left(\widehat{G}_{m}\right)} & \leqslant C_{6}^{l n} l^{|\beta|-l} B^{l-\beta_{n}} 2^{m(|\beta|-l)}\left\|D_{i}^{l} \Phi\right\|_{L_{p}\left(\widehat{\Omega}_{m}\right)} \\
& \leqslant C_{7}^{l n} l^{|\beta|-l}(1+M+d)^{l-\beta_{n}} 2^{m(|\beta|-l)}\left\|D_{i}^{l} \Phi\right\|_{L_{p}\left(\widehat{\Omega}_{m}\right)},
\end{aligned}
$$

где $C_{6}=C_{5} \frac{b}{a}$ и $C_{7}=2^{10} \frac{b}{a}\left(\frac{b}{a} n+b+1\right)$ (мы приняли во внимание неравенство (11)), откуда и следует (10).

6.2. Оценки $L_{p}$-нормы производных продолженной функции. Пусть $-\infty \leqslant a_{i}<c_{i}<d_{i}<b_{i} \leqslant+\infty, i=1, \ldots, n-1, a_{n} \geqslant-\infty$; если $a_{i}=-\infty$ $\left(b_{i}=+\infty\right)$, то $c_{i} \geqslant-\infty\left(d_{i} \leqslant+\infty\right)$. Наряду с множеством $\Omega$, определяемым условиями (1)-(3), рассмотрим множество

$$
\mathscr{E}=\left\{x \in \mathbb{R}^{n}: \quad c_{i}<x_{i}<d_{i}, \quad i=1, \ldots, n-1 ; \quad a_{n}<x_{n}<\varphi(\bar{x})+H\right\},
$$

где $H=2^{-m_{0}-1}$, а $m_{0} \in \mathbb{Z}$.

Положим $G=\mathscr{E} \backslash \bar{\Omega}$ и для $m \in \mathbb{Z}$ (см. рис. 3$)$

$$
\begin{aligned}
& G_{m}=\left\{x \in G, \quad c_{i}<x_{i}<d_{i}, \quad i=1, \ldots, n-1,\right. \\
& \left.\quad 2^{-m-1} \leqslant x_{n}-\varphi(\bar{x})<2^{-m}\right\}, \quad m \geqslant m_{0}-1, \\
& \widetilde{G}_{m}=G_{m-1} \cup G_{m} \cup G_{m+1}, \quad m \geqslant m_{0} .
\end{aligned}
$$

Отметим, что

$$
G=\bigcup_{m=m_{0}+1}^{\infty} G_{m}
$$

Лемма 12. Существует число $C_{8}>0$ такое, что для любьх $n, l \in \mathbb{N}$ существует последовательность неотрицательных функций $\psi_{m} \in C^{\infty}\left(\mathbb{R}^{n}\right)$, $m \in \mathbb{Z}, \quad m \geqslant m_{0}$ (зависящих от $\left.m, n, l, \varphi\right)$, удовлетворяющих следующим условиям:

1) $\sum_{m=m_{0}}^{\infty} \psi_{m}(x)= \begin{cases}1, & x \in G \\ 0, & x \in \Omega\end{cases}$

2) $\bigcup_{m=m_{0}+1}^{\infty} \operatorname{supp} \psi_{m} \subset G$,

причем кратность покрытия $\kappa\left(\left\{\operatorname{supp} \psi_{m}\right\}_{m \in \mathbb{Z}}\right)=2$;

3) $G_{m} \subset \operatorname{supp} \psi_{m} \subset \widetilde{G}_{m}, \quad m \geqslant m_{0}$;

4) $\forall \alpha \in \mathbb{N}_{0}^{n}:|\alpha| \leqslant l u \forall x \in \mathbb{R}^{n}$

$$
\left|\left(D^{\alpha} \psi_{m}\right)(x)\right| \leqslant\left[C_{8}^{n}(1+M) l 2^{m}\right]^{|\alpha|} .
$$




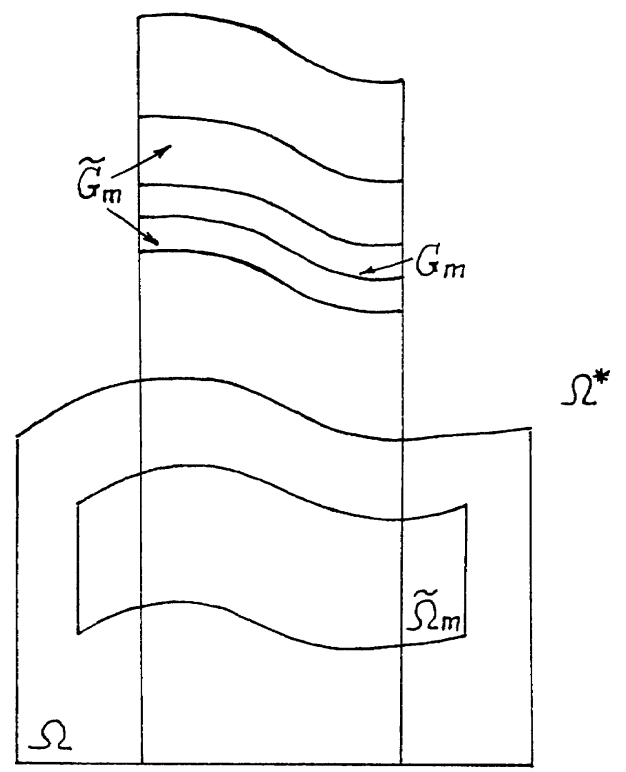

Рис. 3

ДокАЗАТЕЛЬСТво. Продолжим функцию $\varphi$ с параллелепипеда $\left\{a_{i}<x_{i}<b_{i}\right.$, $i=1, \ldots, n-1\}$ на $\mathbb{R}^{n-1}$ с сохранением константы Липшица и обозначим продолженную функцию через $\varphi^{*}$.

Рассмотрим множества

$$
\begin{gathered}
\Omega^{*}=\left\{x \in \mathbb{R}^{n}: x_{n}<\varphi^{*}(\bar{x})\right\}, \\
G^{*}=\mathbb{R}^{n} \backslash \bar{\Omega}^{*}, \\
G_{m}^{*}=\left\{x \in G^{*}: \quad \varphi^{*}(\bar{x})+2^{-m-1} \leqslant x_{n}<\varphi^{*}(\bar{x})+2^{-m}\right\}, \quad m \in \mathbb{Z} .
\end{gathered}
$$

Пользуясь леммой 7 , построим разбиение единицы $\psi_{m} \in C^{\infty}\left(\mathbb{R}^{n}\right), m \in \mathbb{Z}$. Тогда функции $\psi_{m}, m \geqslant m_{0}$, удовлетворяют требованиям леммы 12 .

Пусть $f \in W_{p}^{l}(\Omega)$. Положим $A=200(M n+1)$ и выберем число $m_{0} \in \mathbb{Z}$ так, чтобы

$$
20 A 2^{-m_{0}}<\varepsilon, \quad 8 \cdot 2^{-m_{0}}<\min _{i=1, \ldots, n-1}\left\{c_{i}-a_{i}, b_{i}-d_{i}\right\} .
$$

Для $x \in G$ определим оператор продолжения [6]

$$
\begin{aligned}
(T f)(x) & =\sum_{m=m_{0}}^{+\infty} \psi_{m}(x) \int_{\mathbb{R}^{n}} f\left(\bar{x}-2^{-m} \bar{z}, x_{n}-A 2^{-m} z_{n}\right) \omega(z) d z \\
& \equiv \sum_{m=m_{0}}^{+\infty} \psi_{m}(x) f_{m}(x)
\end{aligned}
$$

где $\omega$ - функция, удовлетворяюшая условиям теоремы 2 . (Если $x \in \Omega$, то $(T f)(x)=$ $f(x)$.) В силу указанного выбора параметров

$$
\text { если } x \in \widetilde{G}_{m}, \quad z \in K, \quad \text { то }\left(\bar{x}-2^{-m} \bar{z}, x_{n}-A 2^{-m} z_{n}\right) \in \widetilde{\Omega}_{m},
$$


где

$$
\begin{aligned}
\widetilde{\Omega}_{m}= & \left\{x \in \mathbb{R}^{n}: \quad a_{i}<x_{i}<b_{i}, \quad i=1, \ldots, n-1,\right. \\
& \left.-8(M n+2 A) 2^{-m}<x_{n}-\varphi(\bar{x}) \leqslant-8(M n+1) 2^{-m}\right\},
\end{aligned}
$$

и значения функции $T f$ на множестве $\widetilde{G}_{m}$ определяются значениями функции $f$ на множестве $\widetilde{\Omega}_{m}$. (Выбор параметров, определяюших множество $\widetilde{\Omega}_{m}$, связан с применением леммы 11 - см. доказательство леммы 13.)

ЛЕмма 13. Оператор T, определенный равенством (27), обладает тем свойством, что при $1 \leqslant p \leqslant+\infty$

$$
T: L_{p}(\Omega) \rightarrow L_{p}(\mathscr{E})
$$

причем существует число $C_{9}>0$ такое, что для любьх $n \in \mathbb{N}, 1 \leqslant p \leqslant+\infty$, $l \in \mathbb{N}$

$$
\|T\|_{L_{p}(\Omega) \rightarrow L_{p}(\mathscr{E})} \leqslant C_{9}^{l n}
$$

ДокаЗАТЕльство. Так как кратность покрытия $\left\{\operatorname{supp} \psi_{m}\right\}_{m=m_{0}}^{+\infty}$ равна 2 , то согласно неравенству Гёльдера при $1 \leqslant p<+\infty$

$$
\begin{aligned}
\|T f\|_{L_{p}(G)}^{p} & \leqslant 2^{p-1} \int_{G} \sum_{m=m_{0}}^{+\infty}\left|\psi_{m}(x)\right|^{p}\left|f_{m}(x)\right|^{p} d x \\
& =2^{p-1} \sum_{m=m_{0}}^{+\infty} \int_{\widetilde{G}_{m}}\left|\psi_{m}(x)\right|^{p}\left|f_{m}(x)\right|^{p} d x \leqslant 2^{p} \sum_{m=m_{0}}^{+\infty}\left\|f_{m}\right\|_{L_{p}\left(\widetilde{G}_{m}\right)}^{p}
\end{aligned}
$$

Положим в лемме $11 a=\frac{1}{4}, b=2, B=A, d=M n+1, c=8(M n+2 A)$; тогда согласно (26) $\widetilde{G}_{m} \subset \widehat{G}_{m}$, а $\widehat{\Omega}_{m}=\widetilde{\Omega}_{m}$, следовательно, согласно неравенству (9)

$$
\|T f\|_{L_{p}(G)} \leqslant 2 C_{2}^{\ln }\left(\sum_{m=m_{0}}^{+\infty}\|f\|_{L_{p}\left(\widetilde{\Omega}_{m}\right)}^{p}\right)^{\frac{1}{p}} \leqslant 2 C_{2}^{\ln } \kappa^{\frac{1}{p}}\|f\|_{L_{p}(\Omega)}
$$

где $\kappa$ - кратность покрытия $\left\{\widetilde{\Omega}_{m}\right\}_{m=m_{0}}^{\infty}(\kappa \leqslant 500-$ см. [6]), откуда и следует (30). Рассуждения при $p=+\infty$ аналогичны. Оператор $T$, определенный равенством (27) с $m_{0} \in \mathbb{N}$, обладает следующим свойством.

Лемма 14. Существует число $C_{10}>0$ такое, что для любых $n \in \mathbb{N}$, $1 \leqslant p \leqslant+\infty, \quad l \in \mathbb{N}, \quad \alpha \in \mathbb{N}_{0}^{n}:|\alpha| \leqslant l, u$ для любых $f \in C^{\infty}(\bar{\Omega})$

$$
\left\|D^{\alpha}(T f)\right\|_{L_{p}(\mathscr{E})} \leqslant C_{10}^{l n}(1+M)^{2 l}\left(\left\|D^{\alpha} f\right\|_{L_{p}(\Omega)}+\|f\|_{w_{p}^{l, \ldots, l}(\Omega)}\right) .
$$


ДокАЗАТЕЛЬСТво. По формуле Ньютона-Лейбница

$$
D^{\alpha}(T f)(x)=\sum_{0 \leqslant \beta \leqslant \alpha} C_{\alpha}^{\beta} \sum_{m=m_{0}}^{+\infty} D^{\alpha-\beta} \psi_{m}(x) D^{\beta} f_{m}(x) .
$$

Обозначим

$$
I_{\alpha \beta}(x)=\sum_{m=m_{0}}^{+\infty} D^{\alpha-\beta} \psi_{m}(x) D^{\beta} f_{m}(x)
$$

Тогда

$$
\left\|D^{\alpha}(T f)\right\|_{L_{p}(G)} \leqslant \sum_{0 \leqslant \beta \leqslant \alpha} C_{\alpha}^{\beta} K_{\alpha \beta}^{\frac{1}{p}},
$$

где $K_{\alpha \beta}=\left\|I_{\alpha \beta}\right\|_{L_{p}(G)}^{p}$. Согласно неравенству (9) из леммы 11 , как и при доказательстве леммы 13 ,

$$
K_{\alpha \alpha}^{\frac{1}{p}} \leqslant C_{9}^{l n}\left\|D^{\alpha} f\right\|_{L_{p}(\Omega)}
$$

Пусть теперь $\beta \neq \alpha(\beta \leqslant \alpha)$. Согласно лемме 12

$$
\sum_{m=m_{0}}^{+\infty} D^{\alpha-\beta} \psi_{m}(x)=0, \quad x \in G .
$$

Отсюда следует, что если $F_{\beta}$ - функция из леммы 11 (напомним, что $F_{\beta}$ не зависит от $m$ ), то

$$
I_{\alpha \beta}(x)=\sum_{m=m_{0}}^{+\infty} D^{\alpha-\beta} \psi_{m}(x)\left[\left(D^{\beta} f_{m}\right)(x)-F_{\beta}(x)\right], \quad x \in G,
$$

и, как при доказательстве леммы 13,

$$
K_{\alpha \beta} \leqslant 2^{p} \sum_{m=m_{0}}^{+\infty}\left\|D^{\alpha-\beta} \psi_{m}\left(D^{\beta} f_{m}-F_{\beta}\right)\right\|_{L_{p}\left(\widetilde{G}_{m}\right)}^{p} .
$$

Согласно неравенству (25)

$$
\left|\left(D^{\alpha-\beta} \psi_{m}\right)(x)\right| \leqslant\left(C_{8}^{n}(1+M)\right)^{|\alpha|-|\beta|} l^{|\alpha|-|\beta|} 2^{m(|\alpha|-|\beta|)},
$$

где $C_{8}>0$ - абсолютная постоянная. Следовательно,

$$
K_{\alpha \beta} \leqslant C_{10}^{n l p}(1+M)^{(|\alpha|-|\beta|) p} l^{(|\alpha|-|\beta|) p} \sum_{m=m_{0}}^{+\infty} 2^{m(|\alpha|-|\beta|) p}\left\|D^{\beta} f_{m}-F_{\beta}\right\|_{L_{p}\left(\widetilde{G}_{m}\right)}^{p},
$$

где $C_{10}>0$ - абсолютная постоянная.

Применяя теперь неравенство (10) из леммы 11 (с теми же $a, b, B, d, c$, что и при доказательстве леммы 13), получим

$$
\begin{aligned}
K_{\alpha \beta}^{\frac{1}{p}} & \leqslant\left[\left(C_{3} C_{10}\right)^{n l p}(1+M)^{(l+|\alpha|-|\beta|) p} l^{(|\alpha|-l) p} \sum_{m=m_{0}}^{+\infty} 2^{m(|\alpha|-l) p}\|f\|_{w_{p}^{l, \ldots, l}\left(\widetilde{\Omega}_{m}\right)}^{p}\right]^{\frac{1}{p}} \\
& \leqslant C_{11}^{n l}(1+M)^{2 l}\|f\|_{w_{p}^{l, \ldots, l}(\Omega)},
\end{aligned}
$$

где $C_{11}>0$ - абсолютная постоянная.

Искомое неравенство (31) следует из (32), (33) и (34). 
ЗАмЕчаниЕ. Если в определении оператора $T m_{0} \leqslant 0$ и $|\alpha|=l$, то неравенство (31) остается в силе, поскольку при выводе неравенства (33) знак $m_{0}$ не играет роли при любых $\alpha \mathrm{c}|\alpha| \leqslant l$, а при выводе неравенства (34) при $|\alpha|=l$ (в отличие от случая $|\alpha|<l$ ) константа также не зависит от $m_{0}$.

Лемма 15. Оператор T, определенный равенством (27), обладает тем свойством, что при $1 \leqslant p \leqslant+\infty, l \in \mathbb{N}$

$$
T: w_{p}^{l}(\Omega) \rightarrow w_{p}^{l}(\mathscr{E}),
$$

причем существует число $C_{12}>0$ такое, что для любых $n \in \mathbb{N}, 1 \leqslant p \leqslant+\infty$, $l \in \mathbb{N}$

$$
\|T\|_{w_{p}^{l}(\Omega) \rightarrow w_{p}^{l}(\mathscr{E})} \leqslant C_{12}^{l n}(1+M)^{2 l} .
$$

ДокАЗАТЕЛЬСтво. Доказательство следует из неравенства (31) и сделанного вьше замечания (предельньй переход показывает, что последнее неравенство справедливо и для любых функций из пространства $\left.w_{p}^{l}(\Omega)\right)$.

Пусть теперь

$$
\Omega=\left\{x \in \mathbb{R}^{n}: \quad \bar{x} \in \mathbb{R}^{n-1}, \quad-\infty<x_{n}<\varphi(\bar{x})\right\},
$$

где $\varphi$ удовлетворяет условию Липшища с постоянной $M$.

Лемма 16. Оператор продолжения $T$, определенный равенством (27) с $m_{0}=-\left(3+\left[\log _{2} l^{l}\right]\right)$, обладает тем свойством, что при $1 \leqslant p \leqslant+\infty, \quad l \in \mathbb{N}$

$$
T: L_{p}(\Omega) \rightarrow L_{p}\left(\Omega^{\delta}\right), \quad T: w_{p}^{l}(\Omega) \rightarrow w_{p}^{l}\left(\Omega^{\delta}\right),
$$

где $\delta=\frac{l^{l}}{1+M}$, причем существует число $C_{13}>0$ такое, что для любых $n \in \mathbb{N}$, $1 \leqslant p \leqslant+\infty, \quad l \in \mathbb{N}$

$$
\begin{aligned}
& \|T\|_{L_{p}(\Omega) \rightarrow L_{p}\left(\Omega^{\delta}\right)} \leqslant C_{13}^{l n}, \\
& \|T\|_{w_{p}^{l}(\Omega) \rightarrow w_{p}^{l}\left(\Omega^{\delta}\right)} \leqslant C_{13}^{l n}(1+M)^{2 l} .
\end{aligned}
$$

ДоКАЗАТЕЛЬСТво. Из [6] известно, что при $x \in G=\bigcup_{m=m_{0}+1}^{+\infty} G_{m}$ справедливы неравенства

$$
\frac{x_{n}-\varphi(\bar{x})}{1+M} \leqslant \varrho(x, \partial \Omega)<x_{n}-\varphi(\bar{x})
$$

Отсюда следует

$$
\delta=\frac{l^{l}}{1+M} \leqslant \frac{2^{-m_{0}-2}}{1+M}<\frac{x_{n}-\varphi(\bar{x})}{1+M} \leqslant \varrho(x, \partial \Omega),
$$

значит, $G \supset \Omega^{\delta}$, и тем самым оператор $T$ продолжает функции с $\Omega$ на $\Omega^{\delta}$.

Теперь утверждение леммы 16 следует из лемм 13,14 и замечания к лемме 14. 


\section{§7. Двусторонняя оценка минимальной нормы операторов продолжения для открытых множеств с границей класса Lip 1}

ОПРЕДЕЛЕНИЕ 3 . Открытое множество $\Omega \subset \mathbb{R}^{n}$ имеет границу класса Lip 1 , если сушествуют числа $\varepsilon>0, M>0, s \in \mathbb{N}$ и открытые параллелепипеды $V_{j}$, $j=1, \ldots, s$, такие, что выполняются следующие условия:

1) $\partial \Omega \subset \bigcup_{j=1}^{s}\left(V_{j}\right)_{\varepsilon}$

2) существует преобразование $\lambda_{j}(j=1, \ldots, s)$, являющееся суперпозищией поворотов и отражений, такое, что

$$
\begin{array}{r}
\lambda\left(V_{j}\right)=\left\{x \in \mathbb{R}^{n}: a_{i j}<x_{i}<b_{i j}, \quad i=1, \ldots, n\right\}, \\
\text { где }-\infty \leqslant a_{i j}<b_{i j} \leqslant+\infty, i=1, \ldots, n, j=1, \ldots, s, \text { и } \\
\lambda_{j}\left(\Omega \cap V_{j}\right)=\left\{x \in \mathbb{R}^{n}: \bar{x} \in V_{j}^{*}, \quad a_{n j}<x_{n}<\varphi_{j}(\bar{x})\right\},
\end{array}
$$

где $V_{j}^{*}=\left\{x \in \mathbb{R}^{n-1}: a_{i j}<x_{i}<b_{i j}, i=1, \ldots, n-1\right\}$, причем

$$
\begin{aligned}
\left|\varphi_{j}(\bar{x})-\varphi_{j}(\bar{y})\right| & \leqslant M|\bar{x}-\bar{y}|, & \bar{x}, \bar{y} & \in V_{j}^{*}, \\
a_{n j}+\varepsilon<\varphi_{j}(\bar{x}) & <b_{n j}-\varepsilon, & \bar{x} & \in V_{j}^{*} ;
\end{aligned}
$$

3) если $V_{j} \cap V_{k} \cap \Omega=\varnothing$, то $V_{j} \cap V_{k}=\varnothing$.

Пусть $\Omega$ имеет границу класса $\operatorname{Lip} 1$ с параметрами $s, M, \varepsilon$. В работе [10] строится последовательность открытых множеств $\Omega_{j}, j=1, \ldots, s$, таких, что $\Omega \equiv \Omega_{0} \subset \Omega_{1} \subset \cdots \subset \Omega_{s}$, причем сушествуют $\delta$, зависящее только от $\varepsilon, M$ и $n$, такое, что

$$
\Omega_{s} \supset \Omega^{\delta},
$$

и набор параллелепипедов $\mathscr{U}_{j} \subset V_{j}, j=1, \ldots, n$, обладающих следующими свойствами.

Множество $\Omega_{j} \backslash \Omega_{j-1} \subset \mathscr{U}_{j}$;

$$
\lambda_{j}\left(\mathscr{U}_{j}\right)=\left\{x \in \mathbb{R}^{n}: \quad c_{i j}<x_{i}<d_{i j}, \quad i=1, \ldots, n\right\},
$$

где $a_{i j}<c_{i j}<d_{i j}<b_{i j}, i=1, \ldots, n-1$ (если $a_{i j}=-\infty$, то $c_{i j} \geqslant-\infty$; если $b_{i j}=+\infty$, то $\left.d_{i j} \leqslant+\infty\right) ;$ множество $\lambda_{j}\left(V_{j} \cap \Omega_{j}\right)$ имеет вид

$$
\lambda_{j}\left(V_{j} \cap \Omega_{j}\right)=\left\{x \in \mathbb{R}^{n}: a_{i j}<x_{i}<b_{i j}, \quad i=1, \ldots, n-1, \quad a_{n j}<x_{n}<\widetilde{\varphi}_{j}(\bar{x})\right\},
$$

где функция $\widetilde{\varphi}_{j}$ удовлетворяет условию Липшица с постоянной $M_{j}=M\left(1+2^{j-s}\right)$, $a_{n j}+\varepsilon_{j}<\widetilde{\varphi}_{j}(\bar{x})<b_{n j}-\varepsilon_{j}, \bar{x} \in V_{j}^{*}$, где $\varepsilon_{j}=\varepsilon\left(\frac{3}{4}-2^{j-s-3}\right)$.

Это, в частности, означает, что множества $\Omega_{j}$ имеют границу класса Lip $1 \mathrm{c}$ параметрами $\varepsilon_{j}, M_{j}$ и параллелепипедами $\mathscr{U}_{1}, \ldots, \mathscr{U}_{s}$.

В соответствии с формулой $(21)$ из $\S 6$ определим для $j=1, \ldots, s$ множества $\mathscr{E}_{j}$ равенством

$$
\lambda_{j}\left(\mathscr{E}_{j}\right)=\left\{x \in \mathbb{R}^{n}: \quad c_{i j}<x_{i}<d_{i j}, \quad i=1, \ldots, n-1, \quad a_{n j}<x_{n}<\widetilde{\varphi}_{j}(\bar{x})+H_{j}\right\} .
$$




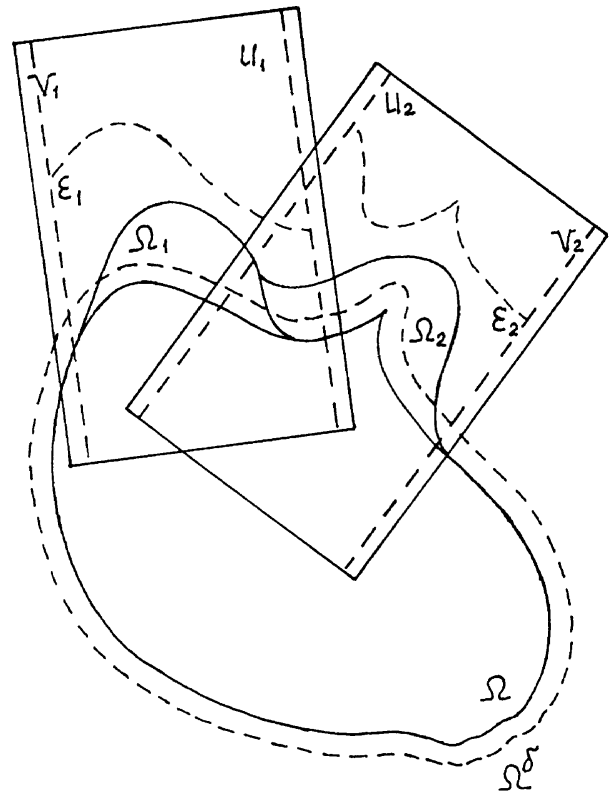

Рис. 4

Построим теперь операторы продолжения $R_{1}, \ldots, R_{s}$. Для функций $f$, заданных на $\Omega_{j-1}\left(\Omega_{0} \equiv \Omega\right)$, положим (см. рис. 4$)$

$$
\left(R_{j} f\right)(x)= \begin{cases}f(x), & x \in \Omega_{j-1}, \\ \left(\Lambda_{j}^{-1} T_{j} \Lambda_{j} f\right)(x), & x \in \Omega_{j} \backslash \Omega_{j-1},\end{cases}
$$

где $\left(\Lambda_{j} g\right)(x)=g\left(\lambda_{j}^{-1} x\right),\left(\Lambda_{j}^{-1} g\right)(x)=g\left(\lambda_{j}(x)\right)$, a $T_{j}$ - оператор, построенньй в $\S 6$ для множества $\lambda_{j}\left(\Omega \cap V_{j}\right)$ :

$$
\left(T_{j} h\right)(x)= \begin{cases}h(x), & x \in \lambda_{j}\left(\Omega_{j-1}\right), \\ \sum_{m=m_{0}}^{\infty} \psi_{m, j}(x) & \\ \times \int_{\mathbb{R}^{n}} h\left(\bar{x}-2^{-m} \bar{z}, x_{n}-A_{j} 2^{-m} z_{n}\right) \omega(z) d z, & x \in \lambda_{j}\left(\mathscr{E}_{j} \backslash \Omega_{j}\right),\end{cases}
$$

где $A_{j}=200\left(M_{j} n+1\right)$, а $m_{0, j}$ выбрано в соответствии с формулой $(26)$ из $\S 6$.

Положим, наконец,

$$
R=R_{s} \ldots R_{1}
$$

(этот оператор продолжает функции, заданные на $\Omega$, на множество $\Omega_{s} \supset \Omega^{\delta}$ ).

ТЕОРема 5. Для любого ограниченного множества $\Omega \subset \mathbb{R}^{n}$ с граничей класса Lip 1 существуют $\delta=\delta(\Omega)>0$ и $C_{1}(\Omega)>0$ такие, что для любых $l \in \mathbb{N}, \quad 1 \leqslant p \leqslant+\infty$

$$
R: L_{p}(\Omega) \rightarrow L_{p}\left(\Omega^{\delta}\right), \quad R: w_{p}^{l}(\Omega) \rightarrow w_{p}^{l}\left(\Omega^{\delta}\right),
$$

причем

$$
\|R\|_{L_{p}(\Omega) \rightarrow L_{p}\left(\Omega^{\delta}\right)} \leqslant C_{1}(\Omega)^{l}, \quad\|R\|_{w_{p}^{l}(\Omega) \rightarrow w_{p}^{l}\left(\Omega^{\delta}\right)} \leqslant C_{1}(\Omega)^{l} .
$$


ЗАмечАниЕ. Утверждение (4) доказано в [10],новым моментом являются оценки (5) для нормы оператора продолжения.

ДокАзАТЕльство. Докажем сначала, что для $j=1, \ldots, s$ существуют такие $B_{j}>0$, зависяшие только от $n, M_{j}$ и $\varepsilon_{j}$, что

$$
\left\|R_{j}\right\|_{w_{p}^{l}\left(\Omega_{j-1}\right) \rightarrow w_{p}^{l}\left(\Omega_{j}\right)} \leqslant B_{j}^{l},
$$

где число $\delta$ выбирается, как описано вьше. Согласно правилам дифференцирования для $\alpha \in \mathbb{N}_{0}^{n}$, для $x \in \Omega_{j} \backslash \Omega_{j-1}$

$$
D^{\alpha}\left(\left(\Lambda_{j}^{-1} g\right)(x)\right)=D^{\alpha}\left(g\left(\lambda_{j}(x)\right)\right)=\sum_{|\beta|=|\alpha|} C_{\alpha \beta}\left(D^{\beta} g\right)\left(\lambda_{j}(x)\right)
$$

и для $x \in \lambda_{j}\left(\mathscr{E}_{j} \backslash \Omega_{j-1}\right)$

$$
D^{\alpha}\left(\left(\Lambda_{j} g\right)(x)\right)=\sum_{|\beta|=|\alpha|} \widetilde{C}_{\alpha \beta}\left(D^{\beta} g\right)\left(\lambda_{j}^{-1}(x)\right),
$$

где $\left|C_{\alpha \beta}\right|,\left|\widetilde{C}_{\alpha \beta}\right| \leqslant 1$. Поэтому для $f \in \omega_{p}^{l}\left(\Omega_{j-1}\right)$

$$
\begin{aligned}
\left\|R_{j} f\right\|_{w_{p}^{l}\left(\Omega_{j} \backslash \Omega_{j-1}\right)} & =\sum_{|\alpha|=l}\left\|D^{\alpha}\left(R_{j} f\right)\right\|_{L_{p}\left(\Omega_{j} \backslash \Omega_{j-1}\right)} \\
& \leqslant \sum_{|\alpha|=l}\left\|D^{\alpha}\left(\Lambda_{j}^{-1} T_{j} \Lambda_{j} f\right)\right\|_{L_{p}\left(\mathscr{E}_{j} \backslash \Omega_{j-1}\right)} \\
& =\sum_{|\alpha|=l}\left\|\sum_{|\beta|=l} C_{\alpha \beta}\left(D^{\beta} T_{j} \Lambda_{j} f\right)\left(\lambda_{j}(x)\right)\right\|_{L_{p}\left(\mathscr{E}_{j} \backslash \Omega_{j-1}\right)} \\
& \leqslant \sum_{|\alpha|=l|\beta|=l} \sum_{\alpha \beta}\left|C_{\alpha \beta}\right|\left\|D^{\beta}\left(T_{j} \Lambda_{j} f\right)\right\|_{L_{p}\left(\lambda_{j}\left(\mathscr{E}_{j} \backslash \Omega_{j-1}\right)\right)} \\
& \leqslant l^{n}\left\|T_{j} \Lambda_{j} f\right\|_{w_{p}^{l}\left(\lambda_{j}\left(\mathscr{E}_{j} \backslash \Omega_{j-1}\right)\right)}
\end{aligned}
$$

Согласно лемме 15 отсюда следует

$$
\left\|R_{j} f\right\|_{w_{p}^{l}\left(\Omega_{j} \backslash \Omega_{j-1}\right)} \leqslant \widetilde{B}_{j}^{l}\left\|\Lambda_{j} f\right\|_{w_{p}^{l}\left(\lambda_{j}\left(\Omega_{j-1} \cap V_{j}\right)\right)}
$$

где $\widetilde{B}_{j}>0$ зависят только от $n$ и $M_{j}$.

Остается заметить, что, как и выше,

$$
\left\|\Lambda_{j} f\right\|_{w_{p}^{l}\left(\lambda_{j}\left(\Omega_{j-1} \cap V_{j}\right)\right)} \leqslant l^{n}\|f\|_{w_{p}^{l}\left(\Omega_{j-1} \cap V_{j}\right)},
$$

и мы получаем (6).

Наконец,

$$
\|R\|_{w_{p}^{l}(\Omega) \rightarrow w_{p}^{l}\left(\Omega^{\delta}\right)} \leqslant \prod_{j=1}^{s}\left\|R_{j}\right\|_{w_{p}^{l}\left(\Omega_{j-1}\right) \rightarrow w_{p}^{l}\left(\Omega_{j}\right)} \leqslant\left(\prod_{j=1}^{s} A_{j}\right)^{l} .
$$

Для доказательства более простой первой из оценок (5) вместо леммы 15 нужно воспользоваться леммой 13 . 
ТЕОРема 6. 1) Для любого ограниченного открытого множества $\Omega$ с границей класса Lip 1 существуют числа $C_{2}(\Omega)>0$ и $C_{3}(\Omega)>0$ такие, что для любых $l \in \mathbb{N}, \quad 1 \leqslant p \leqslant+\infty$

$$
C_{2}(\Omega)^{l} l^{l} \leqslant \inf _{T: W_{p}^{l}(\Omega) \rightarrow W_{p}^{l}\left(\mathbb{R}^{n}\right)}\|T\| \leqslant C_{3}(\Omega)^{l} l^{l} .
$$

2) Если $\Omega=\left\{x \in \mathbb{R}^{n}: \bar{x} \in \mathbb{R}^{n-1},-\infty<x_{n}<\varphi(\bar{x})\right\}$, әде $\varphi$ удовлетворяет условию Липшица, то существует такое $C_{4}(\Omega)>0$, что для любых $l \in \mathbb{N}$, $1 \leqslant p \leqslant+\infty$

$$
1 \leqslant \inf _{T: W_{p}^{l}(\Omega) \rightarrow W_{p}^{l}\left(\mathbb{R}^{n}\right)}\|T\| \leqslant C_{4}(\Omega)^{l} .
$$

ДокАЗАТЕЛЬСТво. 1. Согласно теореме $1^{\prime}$ для множества $\Omega$ и параметра $\delta=\delta(\Omega)$, определенного в теореме 5 , существует функция $\varphi$ такая, что $\varphi \in$ $C^{\infty}\left(\mathbb{R}^{n}\right)$ и $\varphi \equiv 1$ на $\Omega, 0 \leqslant \varphi \leqslant 1$ на $\Omega^{\delta} \backslash \Omega, \varphi \equiv 0$ вне $\Omega^{\delta}$.

Из теоремы 1 для $\alpha \in \mathbb{N}_{0}^{n}:|\alpha| \leqslant l$ следует неравенство

$$
\left|\left(D^{\alpha} \varphi\right)(x)\right| \leqslant C_{5}^{l} l^{|\alpha|} \delta^{-|\alpha|}, \quad x \in \mathbb{R}^{n},
$$

где $C_{5}$ зависит только от $n$.

Положим для $f \in W_{p}^{l}(\Omega)$

$$
(S f)(x)=(R f)(x) \varphi(x), \quad x \in \mathbb{R}^{n} .
$$

Согласно лемме 13

$$
\|S f\|_{L_{p}\left(\mathbb{R}^{n}\right)} \leqslant C_{5}^{l}\|R f\|_{L_{p}\left(\Omega^{\delta}\right)} \leqslant C_{6}(\Omega)^{l}\|f\|_{L_{p}(\Omega)},
$$

где $C_{6}(\Omega)$ зависит только от $\Omega$.

Для $|\alpha|=l$ получаем (согласно (9) и теоремам 4 и 5 )

$$
\begin{aligned}
\left\|D^{\alpha}(S f)\right\|_{L_{p}\left(\mathbb{R}^{n}\right)} & \leqslant \sum_{0 \leqslant \beta \leqslant \alpha} C_{\alpha}^{\beta}\left\|D^{\alpha-\beta} \varphi D^{\beta}(R f)\right\|_{L_{p}\left(\Omega^{\delta}\right)} \\
& \leqslant C_{7}(\Omega)^{l} \sum_{0 \leqslant \beta \leqslant \alpha} C_{\alpha}^{\beta} l^{l-|\beta|}\left\|D^{\beta}(R f)\right\|_{L_{p}\left(\Omega^{\delta}\right)} \\
& \leqslant C_{8}(\Omega)^{l} \sum_{0 \leqslant \beta \leqslant \alpha} C_{\alpha}^{\beta} l^{l}\|R f\|_{W_{p}^{l}\left(\Omega^{\delta}\right)} \leqslant C_{9}(\Omega)^{l} l^{l}\|f\|_{W_{p}^{l}(\Omega)},
\end{aligned}
$$

где $C_{7}(\Omega), C_{8}(\Omega)$ и $C_{9}(\Omega)$ зависят только от $\Omega$, что и доказывает оценку сверху в неравенстве (7).

2. Для доказательства оценки сверху в неравенстве (8) рассмотрим оператор продолжения $T$, построенный в лемме 16, и положим для $f \in W_{p}^{l}(\Omega)$

$$
(S f)(x)=(T f)(x) \varphi(x)
$$

где функция $\varphi$ определена в п. $1 \mathrm{c} \delta=\frac{l^{l}}{1+M}$. 
Согласно (9) и лемме 15

$$
\|S f\|_{L_{p}\left(\mathbb{R}^{n}\right)} \leqslant C_{10}^{l}\|f\|_{L_{p}(\Omega)},
$$

где $C_{10}$ зависит только от $n$.

Для $|\alpha|=l$ получаем (согласно (9) и теореме 4)

$$
\begin{aligned}
& \left\|D^{\alpha}(S f)\right\|_{L_{p}\left(\mathbb{R}^{n}\right)} \leqslant \sum_{0 \leqslant \beta \leqslant \alpha} C_{\alpha}^{\beta}\left\|D^{\alpha-\beta} \varphi D^{\beta}(T f)\right\|_{L_{p}\left(\Omega^{\delta}\right)} \\
& \quad \leqslant C_{11}^{l} \sum_{0 \leqslant \beta \leqslant \alpha} C_{\alpha}^{\beta}\left(\frac{l^{l}}{1+M}\right)^{-(l-|\beta|)} l^{l-|\beta|}\left\|D^{\beta}(T f)\right\|_{L_{p}\left(\Omega^{\delta}\right)} \\
& \quad \leqslant C_{12}(\Omega)^{l}\left[\sum_{\substack{0 \leqslant \beta \leqslant \alpha \\
\beta \neq \alpha}} C_{\alpha}^{\beta} l^{-l(l-|\beta|)} l^{l-|\beta|}\left\|D^{\beta}(T f)\right\|_{L_{p}\left(\Omega^{\delta}\right)}+\left\|D^{\alpha}(T f)\right\|_{L_{p}\left(\Omega^{\delta}\right)}\right] \\
& \quad \leqslant C_{13}(\Omega)^{l}\left(\left\|D^{\alpha}(T f)\right\|_{L_{p}\left(\Omega^{\delta}\right)}+\sum_{\substack{0 \leqslant \beta \leqslant \alpha \\
\beta \neq \alpha}} C_{\alpha}^{\beta} l^{-l(l-|\beta|)+l-|\beta|}\left\|D^{\beta}(T f)\right\|_{L_{p}\left(\Omega^{\delta}\right)}\right. \\
& \quad \leqslant C_{14}(\Omega)^{l}\|T f\|_{W_{p}^{l}\left(\Omega^{\delta}\right)} \leqslant C_{15}(\Omega)^{l}\|f\|_{W_{p}^{l}(\Omega)},
\end{aligned}
$$

где $C_{11}$ зависит только от $n$, а $C_{12}(\Omega), \ldots, C_{15}(\Omega)$ зависят только от $\Omega$, что и доказывает оценку сверху в неравенстве (8).

3. Докажем оценку снизу (7) (в (8) оценка снизу тривиальна). Из неравенства Колмогорова-Стейна по индукции по $n$ следует, что для $f \in W_{p}^{l}\left(\mathbb{R}^{n}\right)$ при $|\alpha|<l$

$$
\left\|D^{\alpha} f\right\|_{L_{p}\left(\mathbb{R}^{n}\right)} \leqslant\left(\frac{\pi}{2}\right)^{n}\|f\|_{W_{p}^{l}\left(\mathbb{R}^{n}\right)} .
$$

Пусть $T: W_{p}^{l}(\Omega) \rightarrow W_{p}^{l}\left(\mathbb{R}^{n}\right)$ - произвольный оператор продолжения. Тогда оценка снизу в неравенстве (7) следует из леммы 6 и теоремы 4 с $|\beta|=l-1$ :

$$
\begin{aligned}
\|T\|_{W_{p}^{l}(\Omega) \rightarrow W_{p}^{l}\left(\mathbb{R}^{n}\right)}\|f\|_{W_{p}^{l}(\Omega)} & \geqslant \frac{C^{*}(\Omega, p, p, l, \beta)}{C^{*}\left(\mathbb{R}^{n}, p, p, l, \beta\right)} \\
& \geqslant\left(\frac{2}{\pi}\right)^{n} C_{5}(n, \mathscr{D})^{l} l^{l-1} \geqslant C_{16}(\Omega)^{l} l^{l},
\end{aligned}
$$

где $C_{16}(\Omega)$ зависит только от $\Omega$. Теорема 6 доказана.

ДокАЗАТЕЛЬСТво п. б) теорем $1,1^{\prime}, 1^{\prime \prime}$. Если предположить противное, то для функции $\varphi$, определенной в доказательстве теоремы 6 , вместо неравенства (9) будет выполняться неравенство

$$
\left|\left(D^{\alpha} \varphi\right)(x)\right| \leqslant C_{5}^{l} l^{\sigma|\alpha|} \delta^{-|\alpha|}, \quad \sigma<1, \quad \forall x \in \mathbb{R}^{n} .
$$

Отсюда следует, что в оценке (11) вместо $l^{l}$ появится множитель $l^{\sigma l}$. Это означает, что

$$
\inf _{T: W_{p}^{l}(\Omega) \rightarrow W_{p}^{l}\left(\mathbb{R}^{n}\right)}\|T\| \leqslant C_{3}(\Omega)^{l} l^{\sigma l},
$$

что противоречит полученной оценке снизу (см. (7)). 


\section{Список литературы}

1. Балашова Г. С. О теоремах продолжения в пространствах бесконечно дифференцируемых функций // Матем. сб. 1982. Т. 118. № 3. С. 371-385.

2. Балашова Г.С. О продолжении бесконечно дифференцируемых функций // Изв. АН СССР. Сер. матем. 1987. Т. 51. №6. С. 1292-1308.

3. Бесов О.В., Ильин В. П., Никольский С. М. Интегральные представления функций и теоремы вложения. М.: Наука, 1975.

4. Буренков В.И. Формула Тейлора и интегральные представления Соболева // Тр. МИАН СССР. 1974. Т. 131. С. 33-38.

5. Буренков В.И. О продолжении функций с сохранением и ухудшением дифференциальных свойств // ДАН СССР. 1975. Т. 224. № 2. С. 269-272.

6. Буренков В.И. Об одном способе продолжения дифференцируемых функций // Тр. МИАН СССР. 1976. Т. 140. С. 27-67.

7. Буренков В. И., Файн Б. Л. О продолжении функций из анизотропных классов с сохранением класса // Тр. МИАН СССР. 1979. Т. 150. С. 52-66.

8. Буренков В.И. О точных постоянных в неравенствах для норм промежуточных производных на конечном интервале // Тр. МИАН СССР. 1980. Т. 156. С. 23-29.

9. Буренков В. И. IX школа по теории операторов в функциональных пространствах: Тез. докладов. Тернополь, 1984. С. 19-20.

10. Буренков В.И. О продолжении функции с сохранением полунормы // Тр. МИАН CCCP. 1985. Т. 172. C. 81-95.

11. Буренков В.И. О точных постоянных в неравенствах для норм промежуточных производных на конечном интервале. II // Тр. МИАН СССР. 1986. Т. 173. С. 38-49.

12. Буренков В.И., Горбунов А. Л. XVI школа по теории операторов в функциональных пространствах: Тез. докладов. Нижний Новгород, 1991. С. 29.

13. Буренков В.И., Горбунов А. Л. Двусторонняя оценка минимальной нормы оператора продолжения для пространства Соболева // Докл. РАН. 1993. Т. 330. №6. С. 680-682.

14. Глушко В. П. Об областях, звездных относительно шара // ДАН СССР. 1962. Т. 144. C. $1215-1216$.

15. Гольдштейн В. М., Решетняк Ю.Г. Введение в теорию функций с обобщенными производными и квазиконформные отображения. М.: Наука, 1983.

16. Горбунов А.Л. XXIX научная конференция факультета физико-математических и естественных наук Российского университета дружбы народов: Тез. докладов. М., 1993. C. 41.

17. Кудрявцев Л.Д. К вопросу о полиномиальных следах // Тр. МИАН СССР. 1983. T. 161. C. $149-157$.

18. Кудрявцев Л. Д. О вариационном методе отыскания обобщенных решений дифференциальных уравнений в функциональных пространствах со степенным весом // Дифференц. уравн. 1983. Т. 19. № 10. С. 1723-1740.

19. Кудрявцев Л. Д., Никольский С. М. Пространства дифференцируемых функций многих переменных и теоремы вложения // Итоги науки и техники. Матем. анализ. Т. 26. М.: ВИНИТИ, 1988. С. 1-157.

20. Мазья В.Г., Поборчий С.В. О продолжении функций из пространств Соболева во внешность и внутрь малой области // Вестн. ЛГУ. 1984. № 7.

21. Митягин Б. М. Аппроксимативная размерность и базисы в ядерных пространствах // УМH. 1964. Т. 16. № 4. С. 63-132.

22. Никольский C. M. К вопросу о неравенствах между частными производными // Тр. МИАН СССР. 1961. Т. 61. С. 147-164.

23. Решетняк Ю. Г. Некоторые интегральные представления дифференцируемых функций // Сиб. матем. журн. 1971. Т. 12. С. 420-432.

24. Соболев $C$. Л. Некоторые применения функционального анализа в математической физике. 1-е изд. Л.: ЛГУ, 1950. 2-е изд. Новосибирск, НГУ, 1962. 3-е изд. М.: Наука, 1988.

25. Файн Б.Л. О продолжении функций с бесконечного цилиндра с ухудшением класса // Тр. МИАН СССР. 1970. Т. 140. С. 277-284. 
26. Яковлев $Г$. Н. О следах функций из пространства $W_{p}^{l}$ на кусочно-гладких поверхностях // Матем. сб. 1967. Т. 74. № 4. С. 526-543.

27. Hestens M. R. Extensions of the range of a differentiable function // Duke Math. J. 1941. V. 8. P. $183-192$.

28. Jones $P . W$. Quasiconformal mappings and extendability of functions in Sobolev spaces. Prepr. Univ. of Chicago, 1980. P. 44.

29. Kalderon A.P. Lebesgues spaces of differentiable functions and distributions // Proc. Symp. Pure Math. 1961. V. 4. P. 33-49.

30. Michlin S. G. Konstanten in einigen Ungleichungen // Teubner-texte zur Matematik. B. 35. Leipzig, 1981.

31. Paukszto $M . V$. On the asymptotic behaviour of the best constant of the extension of functions from the angle // Bulletin de l'Academie Polonaise des Sciences. 1982. V. 30. № 1-2. P. 79-83.

32. Paukszto M. V., Ryll-Nardzewski C. On the Stein Type Extension Operators // Bulletin de l'Academie Polonaise des Sciences. 1982. V. 30. № 7-8.

33. Seeley R. T. Extensions of $C^{\infty}$ functions defined in a half space // Proc. Amer. Math. Soc. 1964. V. 15. P. 625-626.

34. Стейн И. М. Сингулярные интегралы и дифференциальные свойства функций. М.: Мир, 1973.

35. Whitney $H$. Analytic extensions of differentiable functions defined in closed sets // Trans. Amer. Math. Soc. 1934. V. 36. P. 63-89. 